\title{
Une frontière en mouvement : espace public, espace privé dans les cités mayas (Basses Terres centrales et méridionales)
}

Boundaries between public and private spaces in Maya cities of the Central and Southern Lowlands : a spatial object in movement

Frontera entre espacios públicos y espacios privados en las ciudades mayas de las tierras bajas centrales y meridionales : un objeto espacial en movimiento

\section{Damien Bazy}

\section{OpenEdition \\ Journals}

Édition électronique

URL : https://journals.openedition.org/jsa/12843

DOI : 10.4000/jsa. 12843

ISSN : 1957-7842

\section{Éditeur}

Société des américanistes

Édition imprimée

Date de publication : 30 décembre 2013

Pagination : 7-40

ISSN : 0037-9174

Référence électronique

Damien Bazy, "Une frontière en mouvement : espace public, espace privé dans les cités mayas (Basses Terres centrales et méridionales) », Journal de la Société des américanistes [En ligne], 99-2 2013, mis en ligne le 01 janvier 2016, consulté le 03 septembre 2022. URL : http:// journals.openedition.org/jsa/12843; DOI : https://doi.org/10.4000/jsa.12843 


\title{
UNE FRONTIÈRE EN MOUVEMENT : ESPACE PUBLIC, ESPACE PRIVÉ DANS LES CITÉS MAYAS (BASSES TERRES CENTRALES ET MÉRIDIONALES)
}

\author{
Damien BAZY *
}

\begin{abstract}
Cet article traite de l'organisation interne des centres monumentaux des cités mayas classiques à travers une approche innovante. Elle consiste à distinguer les modalités d'interaction spatiale caractérisées par le contact de deux catégories d'espaces, les uns publics et les autres privés, selon une opposition plus efficace que la traditionnelle dichotomie du politico-religieux par rapport au résidentiel. Ce changement de paradigme concernant l'étude de l'urbanisme ouvre le champ d'une série de questions concernant la nature de l'autorité supérieure et les modalités de sa mise en place dans les cités mayas classiques. L'analyse identifie les discontinuités qui séparent les espaces publics des espaces privés, tout autant que les continuités en faisant usage de la notion de « frontière épaisse ». Dans un cadre plus large, les « histoires politiques » produites par l'analyse en synchronie puis en diachronie des plans de cités suffisamment connues contribuent à la recherche sur l'urbanisme classique des cités mayas des Basses Terres centrales et méridionales. [Mots clés : espace public, espace privé, frontière, urbanisme, cités mayas, Basses Terres mayas centrales et méridionales.]
\end{abstract}

Boundaries between public and private spaces in Maya cities of the Central and Southern Lowlands : a spatial object in movement. This article discusses the internal organization of Classic Maya monumental centers through an innovative approach. It aims at distinguishing pattern of spatial interaction characterized by the contact of two categories of spaces, the ones public and the others private, a more efficient opposition than the old politico-religious/residential dichotomy. This change of paradigm in the study of Classic Maya city urbanism opens a broad scope of issues concerning the nature of superior authority and the ways of its establishment. The analysis identifies discontinuities that separate public from private spaces, as well as continuities applying the notion of «thick boundaries". The "political narratives" produced by the synchronic and diachronic analysis of the site maps for selected cities contribute more broadly to the research on Classic urbanism in the Central-Southern Lowland Classic

* UMR 8096, Archéologie des Amériques, Maison René-Ginouvès, 21 allée de l’Université, 92023 Nanterre ; CEMCA, Sierra Leona 330, Lomas de Chapultepec, 11000 México, D.F., México ; School of Anthropology, University of Arizona, Tucson, AZ [damienbazy@hotmail.com].

Journal de la Société des Américanistes, 2013, 99-2, pp. 7-40. C Société des Américanistes. 
Maya cities. [Key words : public space, private space, frontier, urbanism, Maya cities, Central and Southern Maya Lowlands.]

Frontera entre espacios públicos y espacios privados en las ciudades mayas de las tierras bajas centrales y meridionales: un objeto espacial en movimiento. En este artículo se discute la organización interna de los centros monumentales en las ciudades mayas clásicas según un método innovador. Se trata de distinguir las modalidades de interacción espacial caracterizada por el contacto de dos categorías de espacios, unos públicos y otros privados, según una oposición más eficiente que la tradicional dicotomía entre lo político-religioso y lo residencial. Mediante este cambio de paradigma en el estudio del urbanismo surge una variedad de preguntas acerca de la autoridad superior y las modalidades de su aplicación en las ciudades mayas clásicas. El análisis identifica las discontinuidades que separan los espacios públicos de los espacios privados, así como las continuidades mediante el uso de la noción de «frontera espesa ». En un contexto más amplio, las « historias políticas » producidas a partir del análisis en sincronía y luego en diacronía de mapas de ciudades suficientemente conocidas contribuyen a las investigaciones sobre el urbanismo clásico de las ciudades mayas de las tierras bajas centrales y meridionales. [Palabras claves : espacios públicos, espacios privados, frontera, urbanismo, ciudades mayas, tierras bajas centrales y meridionales.]

Cet article aborde la notion de « frontière » en tant que modalité d'interaction spatiale entre deux catégories d'espaces construits diversement accessibles dans les centres des anciennes cités mayas. L'étude archéologique de l'urbanisme maya classique (250-850/900 apr. J.-C.) a depuis longtemps mis l'accent sur les temples-pyramides et les palais articulant les fonctions religieuses et politiques (Harrison 1970), en insistant tantôt sur une séclusion des « épicentres » réservés à une élite liée au roi (Inomata et Houston 2000-2001), tantôt sur la théâtralité de cérémonies politico-religieuses auxquelles aurait assisté toute la communauté (Demarest 1992 ; Inomata 2006). Les analyses étaient freinées par la difficulté à identifier précisément les lieux de résidence, « maison du roi », maisons de la cour royale, bâtiments liés à l'exercice quotidien du pouvoir, quand par ailleurs les fonctions rituelles et cérémonielles étaient aisément repérables dans de nombreux édifices dont les attributs avaient été systématisés (Marcus 1983). Les avancées récentes quant à l'identification archéologique et, dans une certaine mesure, épigraphique de résidences royales et nobles (Andrews et Fash 1992 ; Christie 2003 ; Inomata et Houston 2000-2001 ; McAnany et Plank 2000), a produit une image plus complexe non seulement de ce qui était accessible à tous ou seulement à quelques-uns dans les ensembles monumentaux, mais aussi de ce qui relevait de la collectivité ou répondait plutôt aux intérêts de groupes particuliers. Ce changement de paradigme, qui date au plus tôt des années 1990 avec, en particulier, les découvertes faites à Copan (Fash et al.1992), a ouvert le champ d'une série de questions pour lesquelles de nouveaux outils analytiques sont 
nécessaires. Notre recherche sur l'urbanisme des cités mayas dans les Basses Terres centrales et méridionales (Figure 1) nous a conduit à réorganiser les principes d'analyse des secteurs à architecture monumentale dans ces cités. Posant d'emblée, au moins de façon provisoire à des fins méthodologiques, que la dichotomie public/privé est efficace pour comprendre les centres monumentaux, on a pu établir les critères exclusifs qui discriminent les espaces publics des espaces privés, mais aussi les continuités entre eux. Ce type d'analyse, appliqué à un corpus assez large de cités aux séquences temporelles de construction bien contrôlées, permet de produire une certaine forme de "récit historique » concernant les changements intervenus dans leurs organisations tant architecturales que sociopolitiques (Bazy, Valdés et Arnauld 2010 ; Bazy 2013). Construites indépendamment, ces « histoires archéologiques » viennent compléter l'histoire établie par l'épigraphie, fondée sur le déchiffrement et l'interprétation des inscriptions glyphiques des monuments sculptés de ces mêmes cités.

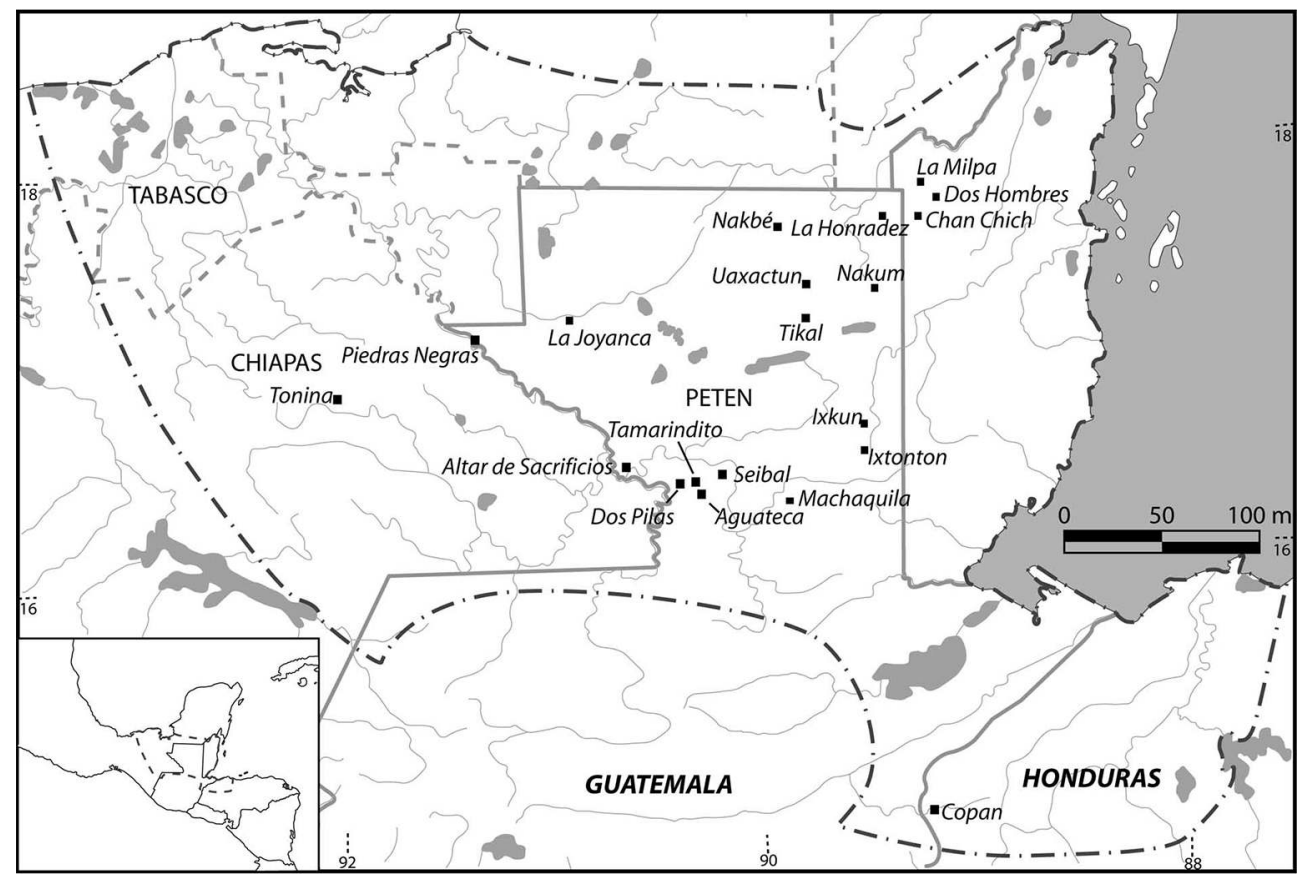

FIG. 1 - Carte de l'aire maya des Basses Terres centrales et méridionales localisant les cités analysées.

Dans les configurations architecturales de ces dernières, il s'agit de concentrer notre attention sur les éléments permettant d'identifier des discontinuités qui distinguent lieux et espaces publics d'autres lieux et espaces privés, et de repérer 
aussi les continuités qui les articulent ou les superposent en les décalant. C'est ici que la notion de "frontière épaisse ${ }^{1}$ constitue un outil dont l'apport est d'autant plus essentiel que la dichotomie privé/public ne va pas de soi appliquée aux conceptions et aux pratiques des habitants mayas de l'époque classique. Sur les notions de public et privé, il faut, par ailleurs, éviter d'en rester à leur opposition qui, appuyée sur le Droit romain, se veut très tranchée dans nos sociétés modernes, tandis qu'elles sont imbriquées dans les sociétés mayas anciennes, ainsi que dans la plupart des sociétés traditionnelles (Inomata et al. 2002, p. 306).

Que désigne le concept de «frontière épaisse »? Selon les principes de la topologie classique, la frontière est une limite linéaire, constituée des bornes qui séparent les intérieurs des extérieurs, un lieu sans épaisseur. Les travaux de Desclés et les applications linguistiques qu'il propose (2002, 2006 ; Desclés et Guentchéva 2010) placent la notion de «frontière épaisse » au cœur de la réflexion concernant les dynamiques de passage entre deux états. L'étude des propriétés topologiques des lieux abstraits tend à montrer que, en réalité, la frontière est un lieu doué d'une épaisseur et en mouvement (Desclés 2012). Cette épaisseur est support de changement dans une certaine continuité. Autrement dit, il s'agit d'un intervalle médiateur entre opposés, d'un processus de passage, d'un intervalle en quelque sorte liminaire où disparaissent les différences (voir Turner 1990 [1969] ; Monod-Becquelin 2012). Cette notion, appliquée à l'architecture, induit, par exemple, que l'espace situé à l'extérieur, à l'entrée d'une résidence, constitue un intervalle potentiel permettant non seulement d'établir l'identité des habitants, mais aussi de convertir l'étranger en un visiteur (Hillier et Hanson 1984, p. 19).

Transposés à l'archéologie maya classique, ces principes analytiques amènent à considérer que des discontinuités correspondent, certes, à des lignes, comme des clôtures ou des murs ou dénivelés, alors que d'autres sont épaisses ou larges, telles les marges, les marches d'un escalier, les seuils d'un édifice ou les dispositifs d'antichambre. Nous mettrons en évidence qu'il y a peu de discontinuités brutales, topologiques, mais plutôt des continuités portant des changements insensibles dès lors que la frontière entre public et privé n'est plus conçue comme une limite, mais comme un lieu en mouvement doté d'une certaine épaisseur.

Dans un premier temps, cette étude reviendra sur les théories récentes qui font des centres des cités mayas des lieux où résidaient ceux qui exerçaient le pouvoir. Puis, on présentera les paramètres permettant de repérer les discontinuités matérielles et conceptuelles entre les catégories duelles d'espaces ici considérés avant d'aborder, à proprement parler, les résultats de notre analyse des relations entre espaces publics et privés dans les cités où les données sont appropriées. La notion de frontière épaisse contribue à l'identification des lieux particuliers à travers lesquels s'opèrent les changements insensibles entre public et privé, ainsi que des relations dynamiques entre ces deux sphères. Elle nous conduira 
notamment à repenser cette dichotomie dans le contexte sociopolitique particulier des sociétés mayas classiques.

\section{La Cité Maya Classique des Basses Terres : THÉORIE ET PRATIQUe}

Les centres monumentaux des cités mayas couvrent une vaste surface (par ex. au maximum 70 ha à Tikal sans les Groupes $\mathrm{O}$, Q et $\mathrm{R}$ au nord; au minimum 5 ha à Dos Pilas), relativement dénuée d'unités résidentielles de bas statut. Il n'y a pas de rues, mais des passages d'une place à l'autre. Cependant, des chaussées maçonnées (sacbeob en maya) relient parfois le centre monumental à des groupes secondaires importants. Le cœur de toutes les cités rassemble la majorité des édifices en pierre taillée reposant sur des soubassements de tailles variables. Aux temples, parfois coiffés d'une crête faîtière, qui surmontent des pyramides hautes de plusieurs dizaines de mètres, s'opposent les palais massifs, tout en longueur. Les palais s'articulent, de proche en proche, autour de cours intérieures et de patios tandis que les pyramides se dressent autour de grandes places, souvent parsemées de stèles et autels. Disposés autour de ces vastes places, des bains de vapeurs, des terrains de jeu de balle, des plates-formes/autels complètent les fonctions de ces grands ensembles. Au centre, l'ordonnancement des édifices maçonnés et voûtés respecte une certaine orthogonalité. Dans la majorité des cités mayas, les ensembles monumentaux du centre et les complexes résidentiels en périphérie adoptent un même azimut.

Faire le choix de fonder des analyses d'espaces urbains architecturés sur la dichotomie entre le public et le privé, fondamentale dans nos sociétés, certainement moins légitimes a priori pour les sociétés mayas anciennes, résulte des avancées notables réalisées récemment dans la compréhension des cités mayas, de leurs configurations et de leur type d'urbanisme (Andrews et Fash 1992 ; Arnauld et Michelet 2004 ; Christie 2003 ; Inomata 1997 ; Inomata et Houston 2000-2001). Sont visés ici leurs secteurs à architecture monumentale qui ont nécessité le travail d'une main d'œuvre nombreuse, dépassant le groupe de parenté. Même si l'ethnographie n'a guère de difficultés à montrer combien les Mayas sont attachés à respecter l'intimité de leurs voisins et à s'assurer qu'on respecte bien la leur (Hanks 1990 ; Pierrebourg et al. 2012), l'opposition du privé et du public ne s'applique pas aisément à l'univers urbain ancien. Elle a cependant des vertus heuristiques dans une première tentative d'analyse, en considérant que le sens le plus général qu'on puisse lui attribuer, le particulier s'opposant au collectif, convient bien aux espaces urbains mayas. Il reflète bien l'opposition entre le « résidentiel » et le « cérémoniel » qui a longtemps été perçue comme ordonnant les ruines des sites. Les petits monticules densément répartis autour des quelques grandes places centrales à pyramides se laissaient bien interpréter, les premiers comme domestiques, les secondes comme cérémonielles. Longtemps, 
l'échelle des constructions et leur abondance relative ont facilement entériné la distinction entre les composantes de l'habitat et celles des grandes cérémonies politico-religieuses. Cette première mise en ordre est vite apparue peu satisfaisante, n'apportant rien de spécifique en termes d'organisation sociopolitique à la connaissance de ces sites labellisés « centres cérémoniels » (voir Becker 1979). Le terme «cérémoniel » a été abandonné au cours des années 1970, celui de « centre » est resté, étant entendu que la " périphérie » demeurait résidentielle. C'est l'épigraphie qui, déchiffrant les inscriptions des monuments sculptés des centres - en somme la signalétique des " centres-villes »-, a conduit à la découverte des royautés mayas qui ont bâti ces édifices, de leurs dynasties, guerres et mariages. On a alors pensé les centres comme des cités au sens politique, dont les édifices monumentaux formaient le décor de la royauté. Les palais ont été identifiés au centre puis, de façon moins prévisible, à la périphérie également (Inomata et Houston 2000-2001).

C'est ici, dans ces édifices résidentiels monumentaux qui ne définissent plus la périphérie ni le centre, pas plus qu'ils ne reflètent simplement le domestique ou le cérémoniel, que s'applique, au moins provisoirement, la dichotomie public/ privé. En dépit de son caractère plus « etic » qu' " emic », son efficacité intéresse l'analyste : le « privé » regroupe sans trop de difficulté le résidentiel, le domestique, l'intime et le particulier ; le « public » accepte pêle-mêle ce qui est cérémoniel, somptuaire, exhibé et collectif. Les notions de visibilité et d'accessibilité jouent bien évidemment un grand rôle dans le fonctionnement de ces catégories appliquées aux grandes maisons royales et nobles (Inomata 2001 ; Moore 1992), lesquelles comportent des cours extérieures, des couloirs, des galeries et des pièces intérieures plus ou moins visibles et accessibles au visiteur étranger. C'est ici que trouve à s'appliquer la notion de frontière épaisse, dans ces espaces complexes de résidences où siégeaient le pouvoir, l'autorité, la force et avec abondance de biens et de personnes. Précisément parce que la royauté dynastique des sociétés mayas classiques ne distinguait pas, ou mal, le patrimoine privé (propriétés de gestion familiale) du domaine public (ressources administrées collectivement; voir Bourdieu 1997 pour les sociétés européennes), les résidences royales et, par imitation, celles des nobles, imbriquaient les deux domaines dans une architecture compliquée, non seulement dans leurs bordures, mais jusque dans leur cœur.

Pour une série de cités mayas sélectionnées dans les Basses Terres centrales et méridionales (Bazy 2013) ont été analysées synchroniquement et diachroniquement les configurations spatiales et architecturales de chaque centre monumental. Par le biais des caractères discriminants tirés de la culture matérielle, en l'occurrence l'architecture, la morphologie des bâtiments, les modalités d'accès, de rassemblement et de circulation, nous avons cherché à comprendre comment les groupes sociaux ainsi distingués, et spatialement circonscrits, étaient articulés au système politico-religieux local, et en réalité le constituaient. 
Contrairement à une tendance généralement implicite consistant à voir du résidentiel là où l'on ne repère pas de politico-religieux, le postulat de départ consiste ici à envisager les composantes résidentielles monumentales comme ayant déterminé les configurations politico-religieuses (et non l'inverse) au cours de l'histoire de la cité. En d'autres termes, l'objectif de cette analyse consiste à identifier la présence et le rôle structurant de factions politiques distinctes et à modéliser les histoires politiques de ces cités parfois occupées depuis le Préclassique jusqu'au Classique terminal (Figure 2), c'est-à-dire depuis 1000 av. J.-C. pour les plus anciennes jusqu'à 950/1000 apr. J.-C. Les progrès de l'épigraphie ont éclairé de nombreux aspects de l'organisation des sociétés mayas classiques. Ils conduisent aujourd'hui à réinterroger l'architecture urbaine pour restituer les organisations récurrentes, leurs évolutions et leurs dynamiques.

Les recherches de Geertz (1980) et Hillier et Hanson (1984) ont inspiré la nôtre. En particulier, dans leur approche théorique et méthodologique de la relation précise entre l'organisation spatiale et la vie sociale, Hillier et Hanson (ibid.) ont montré que l'interface entre la maison et le monde extérieur constitue la différence la plus importante entre un type de cité et un autre. Ils ajoutent que cette interface doit être envisagée dans ses dimensions dynamiques. Dans les Basses Terres mayas, certains travaux ont aidé à l'élaboration de notre méthodologie (Arnauld, Breuil-Martínez et Ponciano A. 2004 ; Fash 2005 ; Martin 2000 ; Nondédéo 2003 ; Valdés 2005). L'analyse des relations entre la Place Principale et le Groupe Guacamaya du site de La Joyanca (Arnauld, Breuil-Martínez et Ponciano A. ibid., pp. 95-124) suggère que les interactions sociopolitiques portées par ces lieux impliquaient des marqueurs sacrés privés et publics dans l'espace de la cité. Les premiers ne concernaient que le groupe de parenté localisé tandis que les derniers « fonctionnaient » pour l'ensemble de la communauté (voir aussi Bazy, Valdés et Arnauld 2010). Arnauld, BreuilMartínez et Ponciano A. ( ibid.) et Martin (2000) proposent qu'il existait, pour un même centre, divers modes d'articulation dans le temps et dans l'espace de ces ensembles construits -, les uns publics, les autres privés.

Ces travaux conduisent à s'interroger sur la méthode la plus appropriée de repérage et de définition de cette éventuelle diversité d'articulations. Combien peut-on en définir? Quelles conclusions en tirer pour établir des organisations sociales spécifiques d'un lieu et d'un moment, et cerner leur sens politique dans des combinaisons spatiales et architecturales, et cela d'un point de vue archéologique parallèle à celui de l'épigraphie?

\section{Frontière conceptuelle entre PUblic/Privé et CRITÈres MATÉriels de DIFFÉRENCIATION}

Pour analyser les frontières entre deux catégories, qu'elles soient d'ordre spatial, temporel ou notionnel, il importe de connaître le contenu de ce qu'elles 


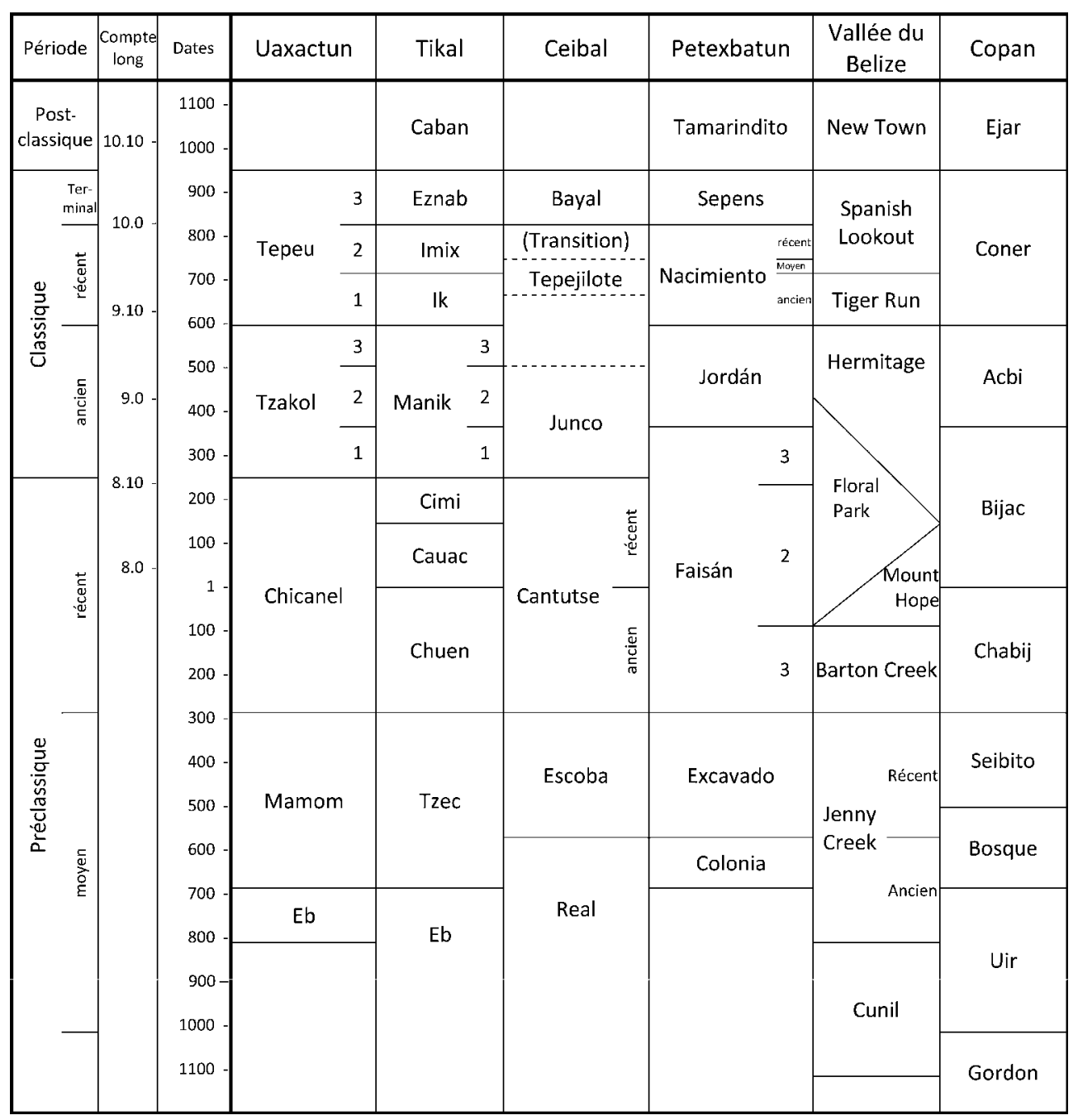

FIG. 2 - Séquences chronologiques des Basses Terres centrales et méridionales fondées sur la céramique, les datations radiocarbone et les dates calendaires des inscriptions mayas. 
délimitent et de bien saisir ce qui est de part et d'autre de la discontinuité. Le rapport dialectique public/privé joue un rôle dans le fonctionnement des lieux construits, depuis la maison jusqu'aux grands espaces collectifs des sociétés aussi bien contemporaines que préhistoriques, et c'est donc un aspect de l'architecture et de l'urbanisme qui dépasse les frontières chrono-culturelles et géographiques. Toutefois, les structures formelles ${ }^{2}$, les habitus ${ }^{3}$ et les pratiques relatives à ces espaces varient évidemment d'une société à l'autre.

Dans les sociétés mayas, les groupes résidentiels ont été construits pour servir les intérêts et illustrer l'identité de la Maison (dans le sens de Lévi-Strauss 1979), c'est-à-dire du groupe social qui l'habitait. En tant que tels, ils peuvent être considérés comme des espaces privés, en particulier leurs autels et sanctuaires domestiques édifiés pour célébrer des rituels propres au groupe local (Figure 3). En revanche, les édifices plus élevés et longs, plus visibles et accessibles qui délimitent de grandes places, ont été construits pour les besoins de la communauté entière, avec de hauts temples qui servaient de cadre pour les cérémonies publiques (Figure 4).

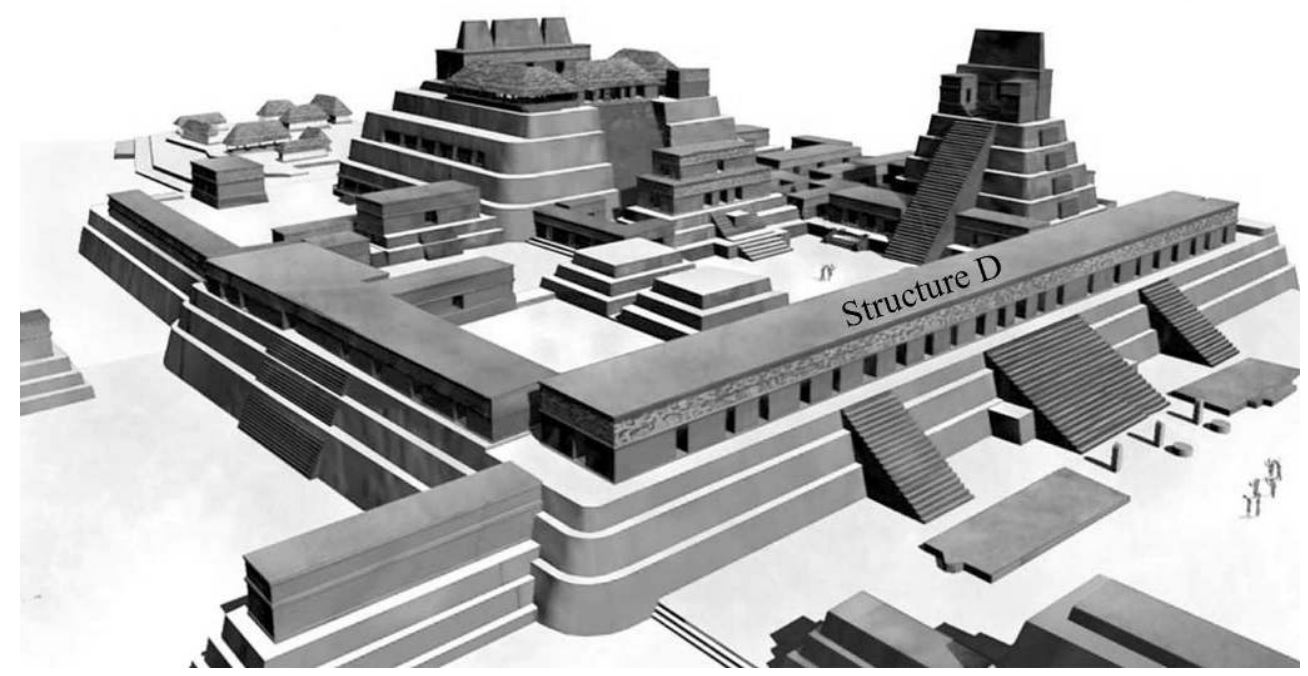

FIG. 3 - Reconstitution de l'Acropole Sud de Nakum au Classique terminal. S'oppose ici au caractère monumental et a priori collectif de ses pyramides, la ségrégation de cet ensemble monumental dont l'accès est contrôlé par une large et longue galerie (Structure D) [d'après Tobar et González 2007, fig. 1, p. 645]. 


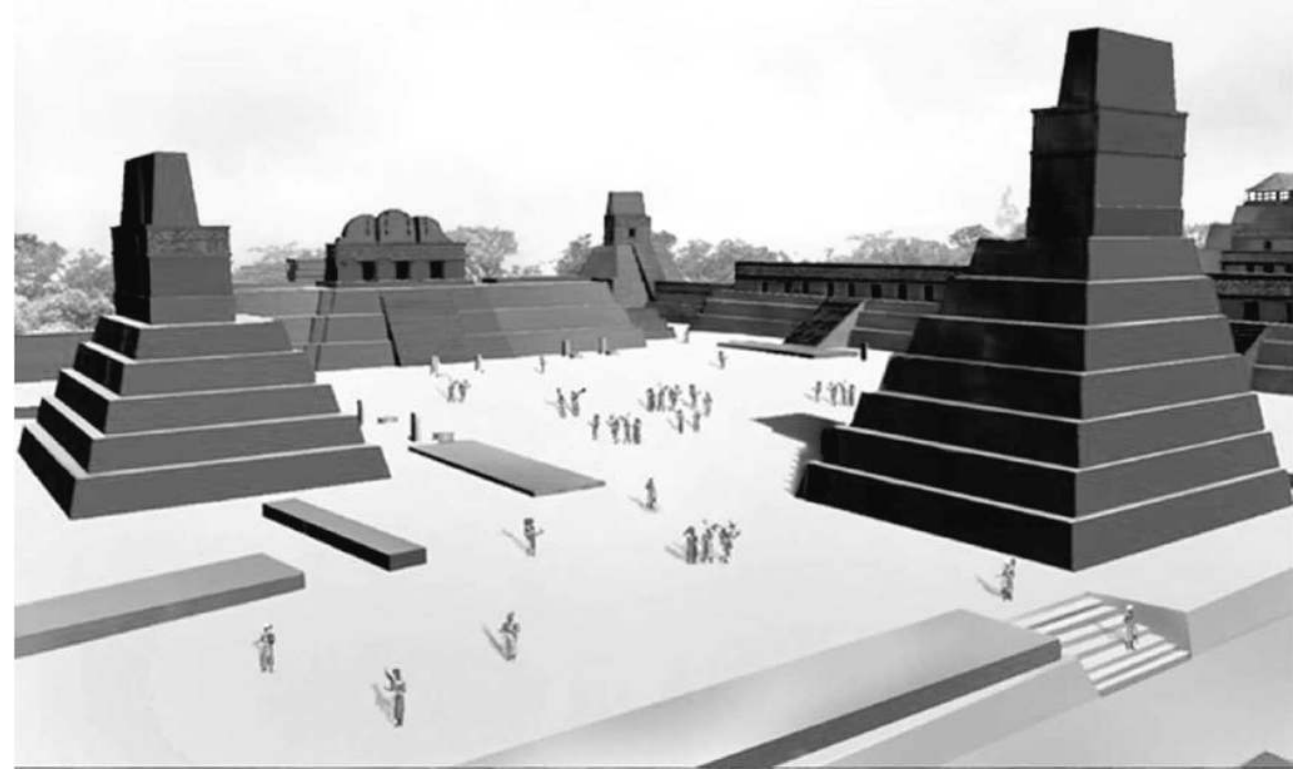

FIG. 4 - Reconstitution de la place centrale de Nakum au Classique terminal dont l'espace aéré et la libre circulation contrastent avec l'espace fragmenté et les accès contrôlés de l'Acropole (voir Figure 3) [d'après Tobar et González 2007, fig. 13, p. 656].

Toutefois, distinguer les espaces privés des espaces publics n'est pas une tâche facile. Des chercheurs ont analysé les agencements spatiaux et les modalités de construction des espaces construits au moyen de divers critères. Tour à tour ont été étudiés le degré d'intimité, ou encore les modalités de la visibilité et de la circulation à l'échelle des édifices (par ex. Arnauld 2001 ; Blanton 1986 ; Inomata 2001, 2006 ; Joyce 2001 ; Liendo 2003 ; Moore 1992, 1996a, 1996b). Or, d'une part, les groupes résidentiels et les édifices publics partagent des agencements spatiaux et des modalités de construction comparables : l'axialité, la centralité, le rôle des portes, la forme générale due à la contrainte de la voûte en encorbellement, sont autant de caractéristiques fondamentales de la maison que l'on retrouve depuis les temples jusqu'aux galeries (Arnauld et Michelet 2010). D'autre part, dans les sociétés mayas anciennes, si le domestique suppose l'intimité, il demande aussi de l'ouverture, de la lumière et des lieux permettant les interactions sociales avec l'extérieur. Inversement, les pyramides supportent des édifices abritant une ou plusieurs pièces de petites dimensions, donc intimes, sombres, non visibles et d'accès restreint (Temple I et II de Tikal), qui n'étaient pourtant pas " privées » pour autant. Ces trois paramètres ainsi évalués de façon 
séparée à l'échelle des édifices pris individuellement ne permettent donc pas de distinguer les lieux dédiés aux affaires publiques de ceux dédiés à la résidence de l'élite. Il faut plutôt les faire interagir simultanément à l'échelle de l'unité-patio et de l'unité-place, en conséquence logique du fonctionnement et de l'agencement de l'habitat maya constitué en unités familiales d'habitat comportant plusieurs résidences et édifices domestiques annexes (cuisines, réserves, oratoires) disposés autour d'une cour (Pierrebourg 1999). Ces ensembles construits des cités mayas se prêtent également à l'analyse de l'ouverture, par opposition à ce qui est compartimenté, ainsi qu'à la distinction entre grands espaces collectifs et petits espaces résidentiels imbriqués. Autrement dit, l'environnement immédiat de l'édifice est discriminant: par ex., s'il est accessible à toute la communauté qui peut y circuler, l'édifice sera certainement plus public que privé puisque le contexte peut suspendre l'intimité et permet des relations d'échanges entre beaucoup d'individus. Inversement, s'il s'agit d'un petit espace résidentiel imbriqué et compartimenté, l'édifice sera certainement plus privé que public puisque peu de gens peuvent y accéder ou, du moins, uniquement des «familiers », membres du groupe de co-résidents. Enfin, sur la base des données archéologiques et par le principe d'abondance, il est depuis longtemps admis que les cités mayas comportent une composante résidentielle majoritaire. Ce paramètre d'abondance ${ }^{4}$, opposé à celui de rareté, peut contribuer à établir la dichotomie entre espace « résidentiel » et espace « communautaire ».

On fait donc, en réalité, interagir la variation dans quatre paramètres combinés : intimité versus exhibition, confidentialité versus visibilité, fermeture versus accessibilité et abondance versus rareté. Ces quatre paramètres sont qualifiés par des critères discriminants, parmi lesquels l'imbrication, opposée à l'ouverture, définit les degrés d'intimité et d'exhibition, et la compartimentation, opposée à la facilité de circulation, contribue à caractériser le degré de fermeture ou d'accessibilité. En outre, d'autres éléments de la culture matérielle peuvent être pris en considération, en particulier la présence ou l'absence de dépotoirs (marquant en principe le domestique privé) ou de stèles (marquant le politique, public). Ainsi caractérisées et différenciées dans des tableaux de données (Figure 5), les unités spatiales, privées ou publiques, sont codées sur les plans des sites et leurs relations peuvent être analysées.

\section{ÉLÉMENTS DE SÉPARATION OU DE RELATION ENTRE UNITÉS SPATIALES}

Les agencements architecturaux, élaborés dans une conjoncture particulière par les acteurs sociaux, reflètent des types d'interactions normalisées, c'est-à-dire l'organisation de la société et, en particulier, au moins certaines de ses institutions sociales. L'espace construit et reconstruit est chargé de sens dès lors que les acteurs sociaux le conçoivent, le construisent, l'utilisent et le modifient en y 


\begin{tabular}{|c|c|c|c|c|c|c|c|c|c|c|c|c|c|c|c|c|}
\hline \multirow{2}{*}{\multicolumn{2}{|c|}{$\begin{array}{c}\text { Paramètres } \\
\text { Critères }\end{array}$}} & \multicolumn{3}{|c|}{ Intimitélextimité } & \multicolumn{2}{|c|}{ Caché/visible } & \multicolumn{7}{|c|}{ Fermeture/accessibilité } & \multicolumn{2}{|c|}{$\begin{array}{l}\text { Marqueurs } \\
\text { d'activités }\end{array}$} & \multirow{3}{*}{ 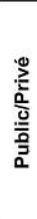 } \\
\hline & & \multirow{2}{*}{$\begin{array}{c}\text { surface } \\
\left(\mathrm{m}^{2}\right)\end{array}$} & \multirow{2}{*}{ 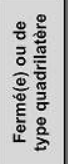 } & \multirow{2}{*}{$\begin{array}{l}\bar{o} \\
\bar{\emptyset} \\
\bar{\emptyset} \\
\overline{0}\end{array}$} & \multirow{2}{*}{ 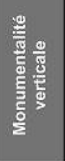 } & \multirow{2}{*}{ 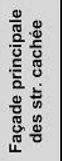 } & \multirow{2}{*}{$\begin{array}{l}\frac{0}{0} \\
\frac{\pi}{5}\end{array}$} & \multirow{2}{*}{  } & \multirow{2}{*}{ 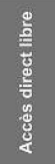 } & \multirow{2}{*}{ 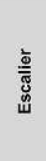 } & \multirow{2}{*}{ 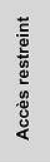 } & \multirow{2}{*}{ 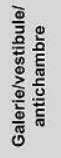 } & \multirow{2}{*}{ 产 } & \multirow{2}{*}{ 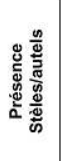 } & \multirow{2}{*}{ 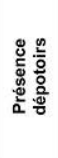 } & \\
\hline Groupe & place/patio & & & & & & & & & & & & & & & \\
\hline \multirow{4}{*}{$\begin{array}{l}\text { Groupe } \\
\text { Ouest }\end{array}$} & $\begin{array}{l}\text { Place } \\
\text { Ouest }\end{array}$ & $7800 \mathrm{~m}^{2}$ & & & $28 \mathrm{~m}$ & & & & & & & & & & & PB \\
\hline & Patio 1 & 280 & & & & & & & & & & & & & & PV \\
\hline & Patio 2 & 230 & & & & & & & & & & & & & & PV \\
\hline & Patio 3 & 240 & & & & & & & & & & & & & & PV \\
\hline $\begin{array}{c}\text { Groupe } \\
\text { Est }\end{array}$ & Place Est & $8120 \mathrm{~m}^{2}$ & & & $12 \mathrm{~m}$ & & & & & & & & & & & PB \\
\hline \multirow{3}{*}{$\begin{array}{c}\text { Groupe } \\
\mathrm{s}\end{array}$} & Patio S-11 & $156 \mathrm{~m}^{2}$ & & & & & & & & & & & & & & PV \\
\hline & Patio S-5 & 250 & & & & & & & & & & & & & & PV \\
\hline & Esplanade & 1250 & & & & & & & & & & & & & & PV \\
\hline
\end{tabular}

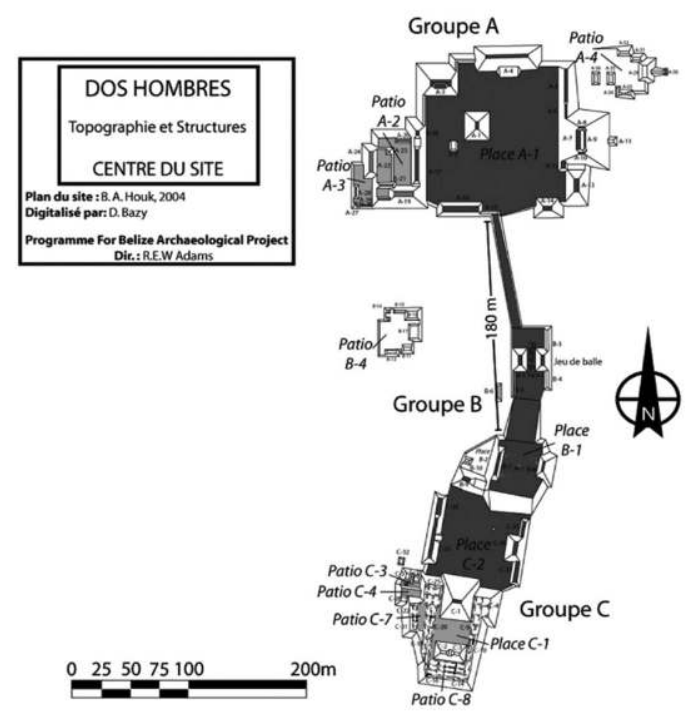

FIG. 5 - Exemple d'usage des quatre paramètres combinés et de leurs critères pour différencier les places publiques des espaces privés dans les ensembles monumentaux de Dos Hombres, Belize [Abréviations : PB : publique ; PV : privé ; NF : secteur non fouillé].

inscrivant la vision d'un monde et de valeurs partagés par tous. De façon certainement simplifiée, mais applicable aux données archéologiques, l'analyse de l'interaction sociale dans l'espace consiste à étudier l'action réciproque entre deux ou plusieurs lieux (Lévy et Lussault 2003). Les modalités d'interaction font 
émerger des dispositifs spatiaux définis par la nature des lieux, mais aussi par la proximité, la séparation et les modalités d'accès. Ces attributs de relations entre lieux construits, accessibles à la seule lecture des relevés topographiques et architecturaux, caractérisent leur degré de séparation, l'épaisseur des limites, des frontières qui les séparent. À La Joyanca, le calcul des distances qui séparent les complexes monumentaux (rang I et II) révèle une fourchette comprise entre $140 \mathrm{~m}$ et $200 \mathrm{~m}$, alors que les groupes à patios qui les composent sont toujours séparés les uns des autres de moins de 50 m (Lemonnier 2006, fig. 7-14 ; voir Peterson et Drennan 2005). Nous avons appliqué cet outil de mesure du degré d'interaction à toutes les cités analysées afin d'identifier les unités sociales ou sociopolitiques, en constatant que la distance d'au moins $140 \mathrm{~m}$ est en effet pertinente de façon assez générale. Bien entendu, chaque cité a dû s'adapter à une situation topographique particulière. Dans certains cas, lorsque la distance n'est pas pertinente, des aspects de la topographie des cités constituent aussi des barrières naturelles entre les composantes spatiales segmentant l'espace urbain : un relief accidenté ou une dépression (marais saisonnier) par exemple. Ainsi, l'Acropole sud et l'Acropole centrale de Tikal ne sont distantes que de $60 \mathrm{~m}$, mais séparées par trois dépressions - le réservoir du temple, celui du palais et le réservoir Escondido -, qui augmentaient la distance perçue entre ces deux complexes architecturaux. Réciproquement deux unités spatiales ayant un côté en commun ou distantes tout au plus de $60 \mathrm{~m}$ sont considérées comme proches. La topographie, les distances réelles et perçues contribuent à définir des limites dont l'épaisseur varie et permettent de repérer les discontinuités ou les continuités qui articulent les complexes monumentaux en interne et entre eux.

Ce mode de mesure de la distance n'est pas le seul possible. Toutes les composantes de l'architecture qui facilitent l'accès à l'intérieur des espaces construits et la communication entre eux sont des «éléments de circulation ». Les «éléments de séparation » interdisent ou limitent l'accès à l'intérieur des ensembles construits. Ces éléments architecturaux réduisent ou augmentent les distances vécues ou perçues, affinent ou épaississent la frontière entre les catégories duelles d'espaces ici considérés. Ainsi, il convient de faire la différence entre escalier et emmarchement. Les escaliers permettent de souligner une différence de hauteurs entre unités, dont l'une « en impose » ainsi à l'autre. Ils marquent bien une sorte de séparation, bien qu'il s'agisse formellement d'un accès. En revanche, l'emmarchement étendu sur le périmètre complet ou sur l'une des façades d'un soubassement facilite la circulation et la station d'une quantité plus importante d'individus sans accentuer visuellement la dimension de la hauteur. Dans certains cas, ces marches servent de tribunes pour le public assemblé autour d'espaces libres dédiés à des événements ou à des cérémonies. Certains édifices servent à contrôler, à orienter la circulation entre un espace et un autre, en particulier ceux qui ont la forme d'une galerie, ou bien d'une pièce unique, sorte d'antichambre ouverte sur la façade antérieure et postérieure. Ces 
escaliers ou emmarchements, seuils ou dispositifs d'antichambre constituent des limites plus ou moins épaisses, des intervalles médiateurs, supports de changement entre l'extérieur et l'intérieur d'un édifice ou d'une cour. C'est tout particulièrement autour de ces éléments architecturaux que se joue la notion de frontière épaisse. Le cas de la Structure D à Nakum (Figures 3 et 4) est particulièrement évocateur de ces espaces liminaires entre lieux publics et privés. En effet, cette longue galerie permet de repérer le changement insensible qui s'opère entre la Place Principale publique (Figure 4) et l'Acropole (Figure 3), espace privé où siégeait le pouvoir. Enfin on doit encore signaler un autre élément de circulation spatiale généralement facile à identifier qui a servi d'élément de cohésion sociale entre groupes sociaux : les chaussées ou sacbeob.

\section{FRONTIÈRES ENTRE PLACE PUBLIQUE ET ESPACES PRIVÉS : DIAGNOSTIC}

Pour évaluer la pertinence de nos outils, une analyse en synchronie des relations entre place publique et espaces privés dans vingt cités mayas des Basses Terres centrales et méridionales de tailles différentes a été effectué (Bazy 2010, chapitre 5). La synchronie signifie que la chronologie garantit ici pour les unités analysées un fonctionnement simultané pendant au moins un temps de l'apogée (généralement le Classique récent), sans tenir compte des détails de leur séquence de construction et de modifications. L'opération a révélé que l'usage de nos quatre paramètres combinés permet de différencier aisément les différents espaces publics et privés dans toutes les cités, à condition de reconnaître, près des unités-patios privés et des unités-places publiques, le caractère ambivalent de certains lieux.

Dans les complexes de palais, des bâtiments ou, plus souvent, des pièces et des dispositifs spatiaux servent de lieux de réunion, de réception des personnes étrangères aux résidents. Ces espaces, qu'on aurait tendance à considérer comme publics, sont imbriqués en fait dans des lieux privés et ont nécessairement été construits en même temps qu'eux ou après eux. Entre dans cette catégorie le dispositif de réception qu'est la pièce centrale, ou la pièce avant dans le cas des dispositions «en tandem» (i.e. avant-arrière) dans les édifices tripartites (Figure 6).

Sous la banquette centrale, était souvent placée la sépulture d'un individu prestigieux soulignant ainsi l'axialité et la centralité du dispositif ${ }^{5}$. Ces lieux, ainsi sacralisés, servaient de scènes de réception pour des visiteurs à l'occasion d'activités politiques et religieuses sous l'égide du chef de Maison (Arnauld et Michelet 2010). Ils étaient aussi, en quelque sorte, des sièges du pouvoir. Un système d'accroche de rideaux au niveau de la porte d'accès permettait, selon qu'ils étaient ouverts ou fermés, de rendre cet espace de réception intime et non visible (privé), ou bien ouvert et visible (public). Cette apparente contra- 


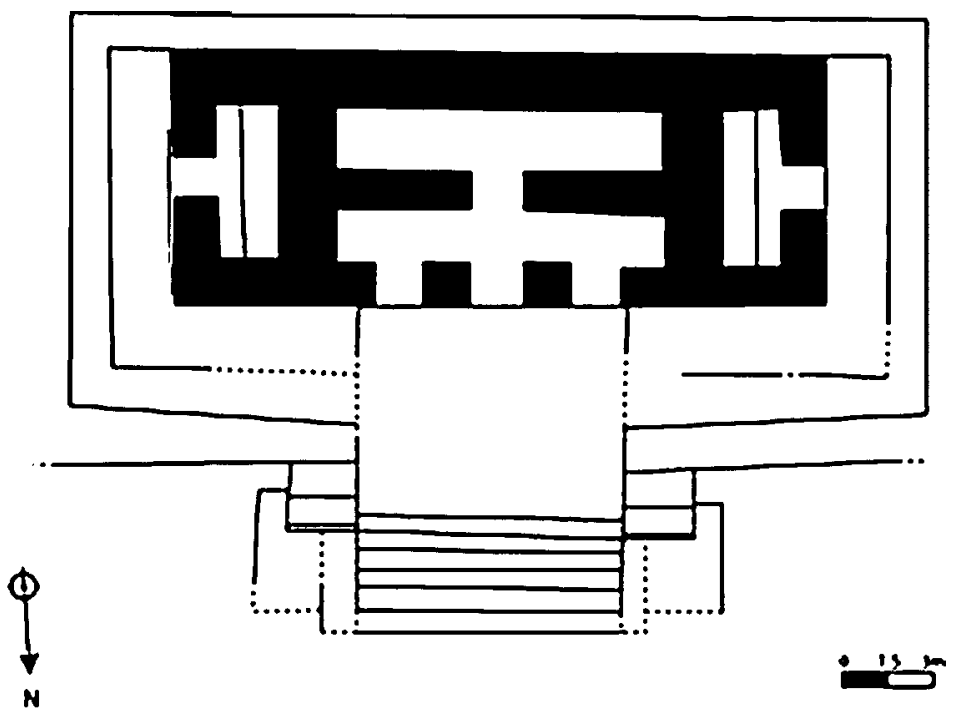

FIG. 6 - Plan tripartite de la Structure G à Nakum, Petén, Guatemala [modifié de Źralka et Hermes 2012, fig. 12, p. 172].

diction fonctionnelle entre l'ordre intime et l'ordre social illustre un cas intéressant de frontière épaisse où s'opère un changement insensible d'état entre les sphères privée et publique. Ces microcosmes politico-rituels semi-publics, servant à des stratégies sociales de pouvoir selon des protocoles qu'on imagine ritualisés, étaient malgré tout plus privés que publics de par leur localisation au sein de groupes résidentiels.

Selon que les pratiques rituelles associées au temple-pyramide sont accomplies à l'intérieur du temple ou sur la plate-forme supérieure de ce dernier s'exprime la dimension privée ou publique de l'édifice. Toutefois, la monumentalité, l'iconographie dédiée aux dieux ou aux ancêtres, l'étroitesse des pièces et la visibilité réduite de l'intérieur depuis l'extérieur affirment ici un caractère moins privé que sacré, que confère le culte des ancêtres du roi ou de toute grande famille. La monumentalité de telles constructions, marquée par exemple par les crêtes faîtières visibles de loin et les messages idéologiques portés par leur iconographie s'adressent tout de même à une large communauté, car le prestige d'un groupe social élitaire ne se construit que face aux autres groupes. Bien que soient attachées à cet élément architectural des affinités et des différences constituées en identités politiques revendiquées par un groupe social particulier à travers le culte aux ancêtres, il s'agit, dans le contexte d'une place, d'un édifice public (ou prétendu tel). En revanche, lorsque celui-ci est érigé au sein d'un patio domestique et familial, il s'agit alors d'un édifice de culte privé (Structures 14 et 15 à Nakum ; Źralka et al. 2007). 
Par ailleurs, signalons qu'au Préclassique récent ont été inventés des microcosmes architecturaux, les complexes triadiques, dont la symbolique et l'iconographie situaient le gouvernant ou chef de maison (ajaw) au centre de l'univers (Valdés 2001). Ces complexes sont ambivalents car, dédiés au particulier, ils montrent tout de même des caractères propres aux places publiques. Ils résultent de grands projets architecturaux rares dans les agglomérations: la hauteur des trois édifices de plan similaire, parfois couronnés d'une crête faîtière, leur confère un caractère ostentatoire, une grande visibilité. Malgré tout, du fait de la hauteur de la plate-forme, de la faible superficie du patio, de leur fermeture progressive par l'ajout de nouvelles constructions au fil des réfections et de leur caractère intime et de leur faible accessibilité, nous leur attribuons une nature plus privée que publique. Certains de ces complexes triadiques ont, de fait, été transformés en complexes palatiaux résidentiels (par ex. Acropole sud de Nakum ; Acropole A-V de Uaxactun).

Ces édifices et lieux ambivalents repérés, il est possible de réfléchir sur la relation entre les espaces construits privés et publics. Le principe général de relation à détecter peut s'énoncer ainsi : «L'expression de l'interaction est la limite, qui peut revêtir des métriques variées » (Lévy et Lussault 2003, p. 522). Les limites les plus repérables sont celles où l'interpénétration des deux espaces est la plus faible, comme dans le cas de frontières matérialisées, ou encore de relation entre espaces à forte opposition de toutes leurs composantes (i.e. échelle, métrique et substance ; ibid.). Inversement, dans les cas d'interpénétration, on a des frontières épaisses dont la spécificité est de ménager des changements dans une certaine continuité. Pour faciliter la lecture et la classification des frontières ou des modalités d'interaction entre place publique et espaces privés, des modèles graphiques ont été élaborés (Figure 7). Les places publiques y apparaissent sous la forme de polygones et les espaces privés de carrés. Ces modèles conservent, autant que possible, la configuration générale de l'établissement. Nous avons montré, à travers de nombreux exemples, que chaque schéma représente un système chargé de sens social et politique, et qu'il évolue en respectant des structures fondamentales propres aux cités mayas (Bazy 2013).

Dans le cas des cités mayas classiques, la relation dialectique entre place publique et espaces privés s'exprime selon diverses modalités. Elle se décline sous la forme de quatre dispositifs spatiaux (Figure 7). Dans le premier dispositif, les unités-patios privées de groupes sociaux distincts sont séparées et distantes de l'unité-place publique (Figure 7-1). Ce dispositif traduit une certaine autonomie de chaque groupe social, ainsi qu'un probable degré de rivalité, de compétition entre tous, car ils pouvaient, ou non, participer aux activités publiques. Dans le second dispositif, une unité-patio privée est séparée de la place publique, mais reliée par le biais d'une chaussée, ou d'une allée réservée (Figure 7-2). Ce dispositif représente un modèle intermédiaire de relation car la séparation est compensée par une chaussée. Une autre entité sociale rivale aurait pu utiliser à ses 


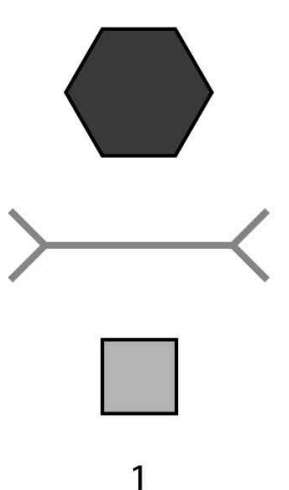

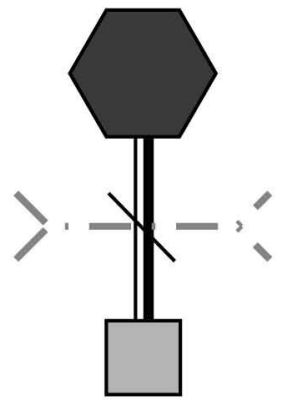

2

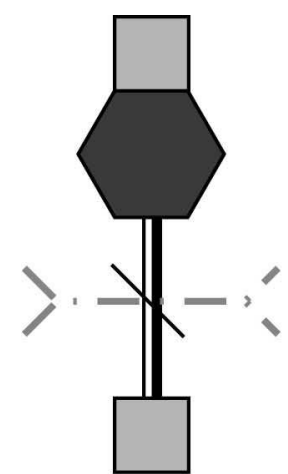

3

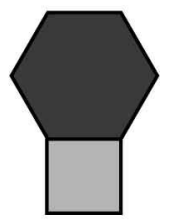

4

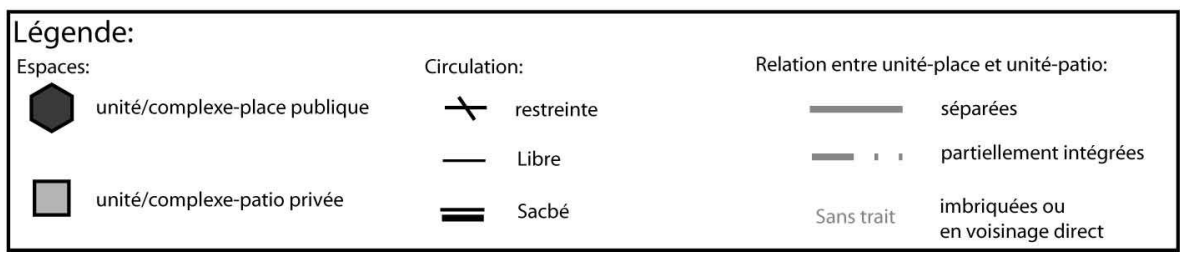

FIG. 7 - Schémas des quatre dispositifs spatiaux correspondant aux modalités de relation entre place publique et espace privé : 1) séparation spatiale ; 2) séparation mais relation par l'intermédiaire d'une chaussée ; 3 ) trois unités spatiales séparées, mais imbriquées par leur relation spatiale, architecturale et chronologique ; 4) union spatiale et architecturale [Bazy 2013, fig. 17, p. 45].

fins, au moins pendant un temps, les lieux publics, mais sans les avoir absorbés dans leur complexe résidentiel privé, ou annexés sur un côté. Le troisième dispositif montre une unité-patio privée séparée, mais reliée par le biais d'une chaussée à la place publique, elle-même incluant une unité-patio privée formant hypothétiquement une antenne de la première dans la place (Figure 7-3). Ces trois unités architecturales ainsi séparées sont pourtant, d'une certaine manière, imbriquées (par ex. Ixtonton, La Joyanca). Enfin, dans le quatrième dispositif, une unité-patio privée est intégrée à la place publique (Figure 7-4). Ce modèle de relation forte implique qu'une entité sociale a pu monopoliser les activités publiques, rituelles et politiques de la communauté (par ex. Acropole centrale et Place Principale à Tikal; Acropole et Place du Groupe Ouest à Piedras Negras).

Ces dispositifs spatiaux, bien que différents d'un point de vue social et politique, se sont développés de manière simultanée ou en séquence dans chacun des vingt sites analysés, de sorte que plusieurs arrangements sont possibles dans le même site. Plusieurs articulations entre ces dispositifs spatiaux apparaissent de manière récurrente dans des cités de rangs hiérarchiques distincts, localisées dans des environnements différents, de sorte que ces articulations ne répondent ni à des règles prédéfinies d'organisation spatiale, ni à un cosmogramme, ni à une 
adaptation au cadre naturel. Au contraire, elles ont été construites par des acteurs sociaux au fort potentiel politique. Autrement dit, lesdites articulations reflètent une situation sociale et politique, un certain rapport de force à un moment donné, et attestent de leur historicité.

Par exemple, à l'apogée de cités aussi différentes et inégales que La Joyanca (600-750/900 apr. J.-C.) et Copán (600-800 apr. J.-C.), la situation implique plusieurs groupes de co-résidents dont les complexes de palais privés segmentent la composante monumentale des différentes agglomérations. Parmi ces groupes, une Maison plus puissante que les autres mobilisait les activités publiques pour affirmer son autorité et assurer la continuité de la dynastie royale. C'est le cas à La Joyanca où l'entité Guacamaya aurait utilisé à ses fins, pendant un temps au moins, les lieux publics sans les absorber dans son complexe (Figure 8).

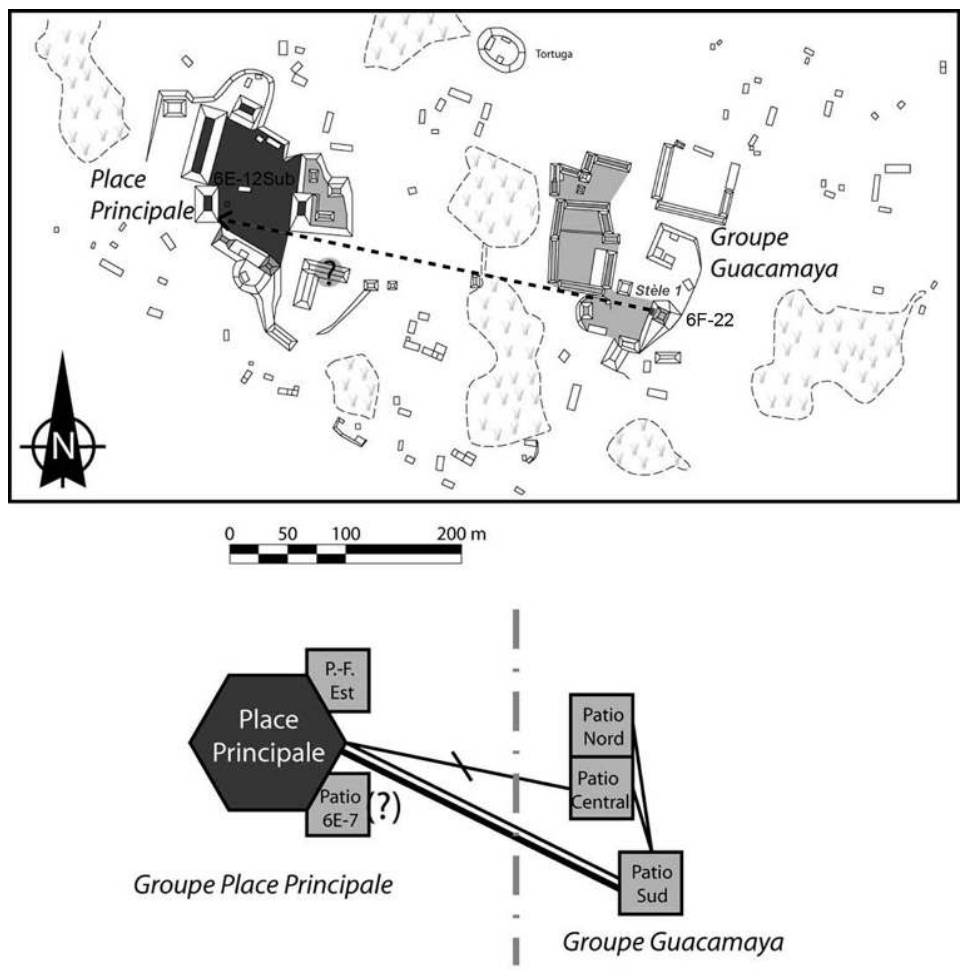

FIG. 8 - Plan et schéma de relation entre la place publique et les espaces privés de la Maison dominante à l'époque d'apogée de la cité de La Joyanca ; en pointillé l'allée-perspective entre l'autel 6F-22 du culte privé de la Maison Guacamaya (à droite, avec sa stèle et ses sépultures) et la Structure 6E-12Sub de la Place Principale. Il n'y a pas vraiment de chaussées construites ; l'allée constituant le seul accès formel à la Place Principale est marquée par l'absence de structure sur l'axe et par le remblaiement ancien formant un passage dans un marais. 

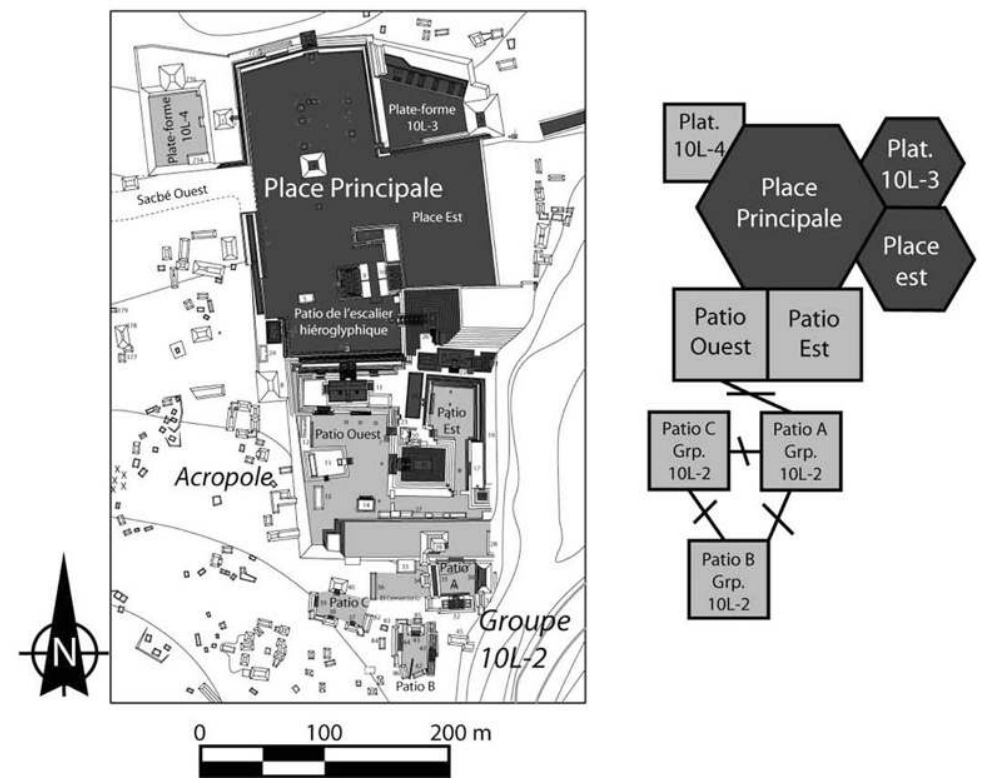

FIG. 9 - Plan et schéma de relation entre la place publique et les espaces privés de la Maison dominante à l'époque d'apogée de la cité de Copán, relation forte entre le complexe palatial 10L-2, l'Acropole et le complexe de places publiques, son jeu de balle et son escalier hiéroglyphique.

En revanche, à Copán, la Maison dominante était parvenue à instaurer un régime dynastique : le complexe de palais du Groupe 10L-2 et l'Acropole sont accolés l'un à l'autre dans le complexe de places publiques du Groupe Principal (Figure 9). Les autres complexes de palais privés, avec leurs temples domestiques édifiés pour célébrer les cultes propres, et leur scène de réception, sont, quant à eux, dispersés à distance des palais royaux et de la place publique.

Certaines de ces entités coopéraient, notamment celles reliées à la place par une chaussée, quand d'autres étaient en compétition entre elles et avec la lignée royale. Dans certains cas, on observe une tendance à la dualité politique (Figure 10) : les résidences de la Maison dominante étaient là imbriquées dans les places publiques, mais une seconde entité séparée disposait d'une forte relation avec les places publiques par le biais d'une chaussée. Si cette seconde entité avait sa propre place publique (Place du Groupe Sud), celle-ci était en ruine à l'époque d'apogée (Webster et Houston 2004, pp. 439-443 ; voir aussi Bazy 2011). Parfois enfin, s'exprime une forte dualité politique faisant s'affronter deux Maisons de haut rang pour l'acquisition de la suprématie et du pouvoir communautaire (Figure 11). 

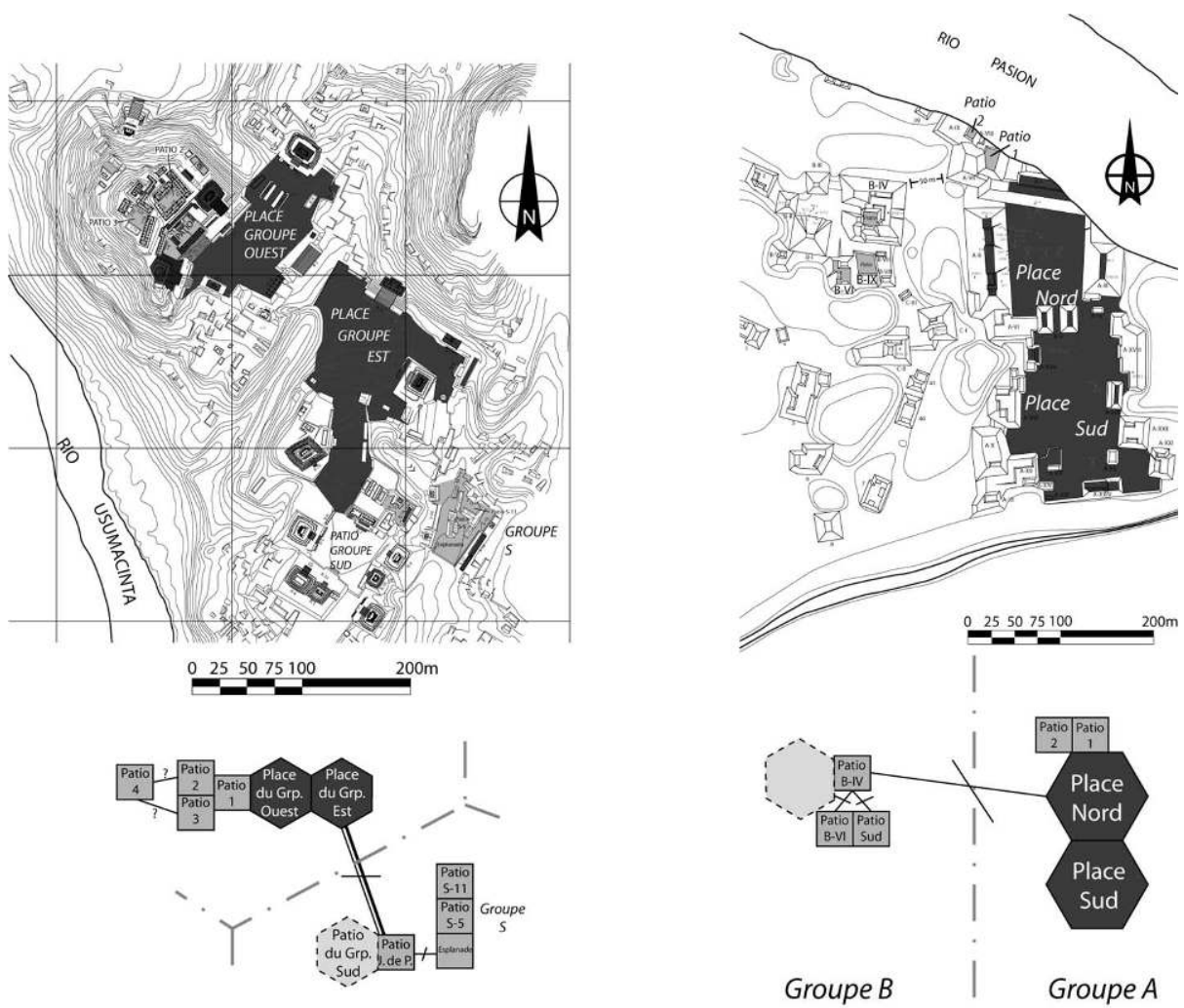

FIG. 10 - Exemples de relation entre place publique et espaces privés exprimant une tendance à la dualité (à gauche, Piedras Negras dont la place publique du Groupe Sud a été abandonnée au Classique ancien); à droite, Altar de Sacrificios dont la place publique du groupe B a été également abandonnée au Classique ancien).

En résumé, l'usage combiné des quatre paramètres retenus permet de repérer aisément les deux catégories d'espaces dans toutes les cités. D'autre part, l'analyse spatiale en synchronie met en lumière des constantes, des schémas récurrents de relations entre sphères publique et privée, et permet de distinguer le palais du roi des grandes places publiques, mais aussi d'autres résidences de groupes alliés ou rivaux de la lignée royale, et ainsi d'esquisser en quelque sorte l'ossature d'un rapport de force entre factions politiques à l'apogée de la cité. 


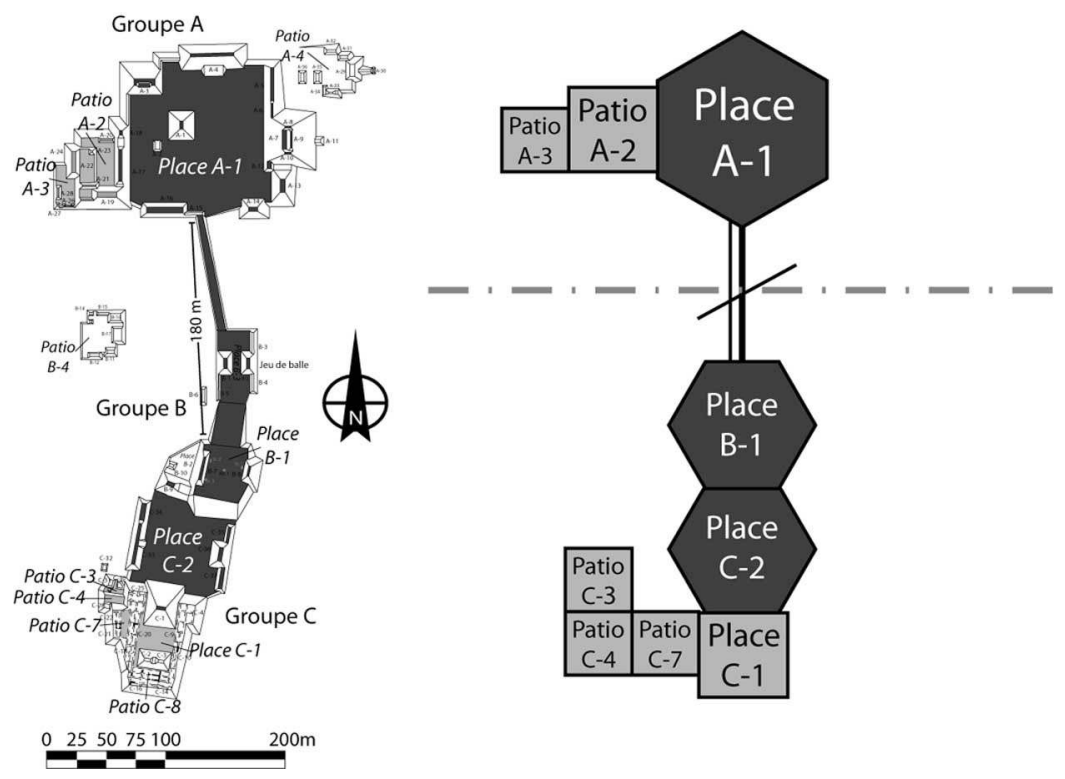

FIG. 11 - Exemple de relation entre place publique et espaces privés exprimant une forte dualité politique (Dos Hombres, Belize).

\section{DISCUSSION}

Dynamique des relations entre espaces publics et espaces privés : une frontière mobile

La transformation des modalités d'interactions, des frontières entre les lieux publics et privés par le biais de changements dans l'architecture et l'espace au cours de l'occupation permet donc de restituer une certaine histoire pour chaque site. Les édifices des centres urbains durent au-delà d'une génération et transmettent la mémoire de configurations politiques successives jusqu'à ce que la construction de nouvelles articulations les altère ou les détruise. Par l'analyse spatiale en diachronie, il s'agit d'approcher les dynamiques de négociations (alliance, mariage, rivalité, autonomie, coopération, absorption) entre factions rivales, leurs stratégies à travers les récits biographiques des lieux (Ashmore 2009), en somme l'histoire politique de la cité.

L'analyse de dix sites dont la séquence de construction est suffisamment bien connue permet de détecter des convergences dans les histoires locales vers un processus d'évolution générale, malgré des variations propres à chaque cité (Figure 12 ; Bazy 2013). Bien entendu préliminaire, ce schéma général devra être 

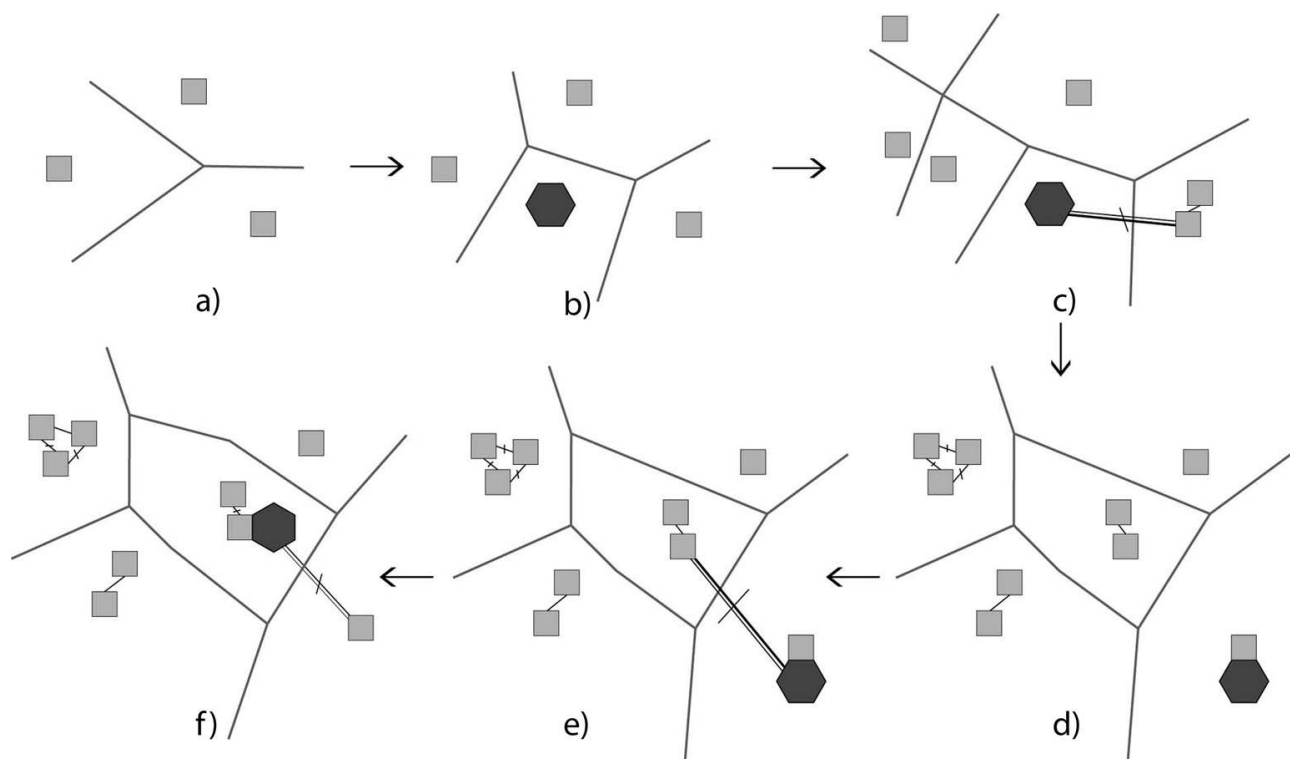

FIG. 12 - Schémas des processus d'évolution générale des cités mayas des Basses Terres centrales et méridionales : a) installation dispersée de plusieurs entités sociales autonomes; b) fondation d'une place publique partagée; c) translation sur la place publique des éléments symboliques consacrant la Maison dominante; d) intégration de la place publique dans les espaces privés de la Maison dominante ou vice versa; e) appropriation de la place publique; f) abandon de l'ancienne place publique et création d'une nouvelle place publique intégrée au complexe de patios privés de la nouvelle Maison dominante.

amendé et amélioré. Il ne faut pas voir à travers le modèle interprétatif développé ici un patron immuable. Il s'agit plus d'une mise en ordre et d'une tentative de trouver des cohérences afin d'orienter idéalement les programmes de fouilles archéologiques, avec l'objectif sous-jacent d'établir des modèles de processus de transformation de la cité par l'observation des héritages, des inerties, des trajectoires et des dynamiques inscrits dans le temps long de l'architecture monumentale, pour définir des hypothèses qui permettent de faire des expérimentations.

Chaque étape du processus répond à une variation limitée des stratégies sociopolitiques mises en œuvre dans l'espace architecturé par les groupes sociaux pour exercer le pouvoir. L'analyse diachronique nous a donc permis d'identifier des systèmes évolutifs de relations entre espaces publics et privés. Selon les stratégies sociopolitiques développées par les uns et par les autres, ces systèmes évoluent en respectant des structures fondamentales propres aux sociétés mayas. Leur traduction dans l'architecture répond aux contraintes de formes, de techniques et de ressources à disposition. 
Au début, dans la première étape de ce processus d'évolution, les vestiges révèlent une installation dispersée de plusieurs entités sociales autonomes (Figure 12a). C'est de loin l'étape la moins connue et la plus problématique. Ces entités se dédiaient à construire peu à peu et à consacrer, par le biais d'activités rituelles privées, la " Maison » physique, lieu central d'un groupe social donné. Dans la deuxième étape, c'est sous l'impulsion de l'une ou plusieurs de ces Maisons fondatrices qu'est définie une place pour des rituels publics collectifs (Figure 12b). En tant qu'instrument de cohésion de la communauté globale, cette place a sans doute représenté un enjeu fondamental lorsqu'un groupe affirmait sa suprématie sur les autres. Dans la troisième étape, en transférant sur la place publique, depuis ses lieux de résidence, les éléments symboliques qui consacraient l'identité du groupe, l'une des Maisons fondatrices, plus puissante que les autres, tentait de mobiliser les activités publiques à son profit pour affirmer son autorité (Figure 12c). Mais c'est seulement dans la quatrième étape, en intégrant la place publique à leurs résidences privées ou vice versa, que les composantes de la Maison perpétuent leur autorité et qu'est instauré le pouvoir dynastique (Figure 12d). Ce processus peut être interrompu à tout moment par l'une ou l'autre des Maisons rivales. C'est en reproduisant les dispositifs spatiaux des deux dernières étapes - i.e., appropriation de la place publique (Figure 12e), création d'une nouvelle place intégrée aux complexes palatiaux privés de la nouvelle autorité (Figure 12f) -, qu'une entité sociale acquiert la possibilité de contester l'autorité et d'instaurer une nouvelle lignée dynastique.

Force est de reconnaître que la frontière entre place publique et espaces privés ne ressemble pas à un mur rigide, inébranlable, érigé une fois pour toutes et solidement gardé. Elle fluctue au gré des intentions des acteurs sociaux au fort potentiel politique. Elle est en mouvement, tel un front pionnier. Chaque action visant à modifier cette frontière a de profondes implications symboliques et sociales. Du moins, c'est ce qu'illustre l'analyse diachronique des relations entre places publiques et espaces privés dans le champ de l'archéologie. En effet, une dynamique bien spécifique des relations entre la sphère publique et la sphère privée fonde le fonctionnement politique des cités mayas : c'est la translation, vers la place publique, des éléments symboliques qui consacrent rituellement la Maison maya en tant que lieu où s'expriment l'identité et les racines ancestrales d'un groupe social particulier. Par ce processus, ces symboles deviennent les instruments de l'intégration politique de toute la communauté et la translation spatiale représente la condition de possibilité d'exercice de l'autorité sur la communauté toute entière. Ce processus implique une continuité entre deux altérités, une frontière épaisse entre « l'ordre proprement politique des pouvoirs publics, doté de sa logique propre (la raison d'État), de ses valeurs autonomes, de son langage spécifique ", et le pouvoir privé de la Maison royale qui « obéit à un mode de gestion et de reproduction patrimoniale » (Bourdieu 1997, p. 63). 


\section{Sacralisation et appropriation : pratiques créatrices de discontinuités}

Ces processus politiques conduisent à reconnaître que l'appréciation juste de la place des frontières entre espaces publics et espaces privés suppose la prise en compte de bien d'autres considérations que la seule mesure des distances spatiales ou les seules limitations matérielles. La matérialité n'est qu'une dimension de la limite.

La sacralisation ou l'appropriation, tacite ou licite, d'un lieu par des rites ancestraux sont des gestes extrêmement «tomogènes » (qui produisent des discontinuités ; Lévy et Lussault 2003, p. 208). Dans la sphère privée, depuis les périodes les plus anciennes, la proximité entre les vivants et les morts revêt une importance particulière (McAnany 1995, 1998). Certains individus sont en effet ensevelis sous le sol des patios ou de la maison. Ces cultes, que chaque Maison peut rendre à ses ancêtres, constituent des rites partagés par tous les membres de la société, mais sont en eux-mêmes une part de l'identité de chaque Maison (Godelier 2004, pp. 107-108). À travers leur maintien depuis le Préclassique jusqu'à nos jours, l'ancienneté et la force des rites de fondation et de clôture sont également manifestes. Les acteurs sociaux devaient perpétuer ces marqueurs matériels du sacré afin de maintenir la Maison, pour assurer la reproduction des relations sociales qui lient les membres d'une entité et les distinguent des autres. Quant aux rituels perpétués dans la sphère publique, dans les cités où l'architecture monumentale de pierre taillée se manifeste précocement, ils sont hérités de croyances agricoles profondément ancrées ou d'une conception du monde partagée par tous. Les lieux sacrés où ces rituels sont réalisés constituent des espaces de la communauté, comunitas ou de communion d'individus égaux (sans statut, nom, fonction, ni pouvoir) qui se soumettent ensemble à l'autorité des rituels ancestraux (Turner 1990 [1969]). Ces lieux publics contribuent à rendre visibles les groupes distincts et représentent une image de la totalité de la communauté. Ils offrent une traduction spatiale, parfois cosmologique, de valeurs, de normes socioculturelles et religieuses. Toutefois, selon Turner (ibid.), la comunitas établie par la réalisation des rites est instable. Il s'agit d'une phase, d'un moment, pas d'une condition permanente. C'est pourquoi tout type d'intervention dans cet espace peut changer sa dynamique, sa composition, ses limites, il peut même parfois en modifier le statut et il peut transformer alors de manière profonde le pacte qui fonde le lien communautaire (Berdoulay et al. 2004, p. 12) et les relations sociales et politiques.

Le statut public de l'espace est, en effet, menacé chaque fois que l'on tente d'en faire un lieu où s'attachent des affinités et des différences constituées en identités politiques. Dans le cas où certaines identités de groupes particuliers cherchent une reconnaissance de leur légitimité, cet espace peut se convertir en l'objet de leur appropriation. Il représente un enjeu fondamental lorsqu'un groupe affirme 
sa suprématie sur les autres. Si la Maison est, chez les Mayas anciens, la matrice des édifices politiques, il est logique que toute faction, qui est de l'ordre du familial (plutôt que du parti politique ou de la société secrète), vise à s'approprier les lieux publics pour les absorber d'une façon ou d'une autre dans sa Maison. Elle ambitionne également de magnifier sa Maison comme lieu public grâce aux mécanismes visant à projeter, vers la place publique, les éléments symboliques qui contribuent à la perpétuation de son patrimoine matériel et symbolique. Cette appropriation des lieux publics se transmettant à travers le réseau des relations familiales conduit à la constitution de véritables dynasties. Les stèles participent également de cette stratégie d'appropriation de la place publique. Les stèles sculptées à l'effigie d'individus de statut royal indiquent aux entités sociales en compétition la propriété d'une place publique.

\section{Langage symbolique et " frontières épaisses " entre lieux publics et privés}

Parmi les éléments symboliques déployés par un groupe social de façon visible pour se distinguer des autres et ainsi assurer sa propre cohésion interne, des lieux, capables de défier le temps et d'incarner de manière durable l'institution sociale de leur "Maison", sont conservés pour l'archéologue. Au même titre que les pratiques rituelles, ces lieux particuliers étaient investis d'une charge fonctionnelle et symbolique probablement dérivée de la conception matérielle, immatérielle et idéelle maya de la Maison. Il s'agit des lieux sacrés dédiés au culte des ancêtres (temples, autels, voire sépultures) et à l'exercice du pouvoir (dispositif de réception) : le temple familial dédié au culte des ancêtres passe du patio à la place sous la forme du temple dynastique (par ex. à Copán, le Temple familial 10L-16 est transféré depuis l'Acropole vers la Place Principale sous la forme de la Pyramide 10L-26 et son escalier hiéroglyphique ; Bazy 2013). L'édifice tripartite avec son dispositif de réception privé se convertit en galerie de type « siège du pouvoir » public (par ex. à Tikal, le dispositif de réception de la Structure 5D-65 est transféré depuis l'Acropole centrale vers la Place Principale sous la forme de la Structure 5D-120 ; ibid.); et, à la transition entre la sphère publique et la sphère privée, le seuil du dispositif de réception transformé en galerie de distribution (au sens architectural) matérialise la frontière entre ces altérités (par ex. Structure D à Nakum). Cette transformation épaissit la distance sociale entre les membres de la Maison imbriquée dans la place publique et les autres Maisons rivales ou alliées.

Construire un lieu dont le caractère sacré réside dans sa capacité à intégrer un groupe particulier à une échelle de plus en plus publique (depuis le rituel sous le sol de la maison, en passant par l'autel central ou l'oratoire construit sur un côté de l'unité d'habitat, jusqu'au temple-dynastique sur une place) illustre, non pas tant une frontière prenant la forme d'une ligne de séparation entre deux altérités, mais plutôt un gradient entre deux unités spatiales, une discontinuité épaisse. Ce 
gradient, allant du privé à ce qui devient de plus en plus public, se rapporte à des capacités proportionnellement accrues de mobilisation sociale de groupes (mesurées par l'architecture monumentale) et donc d'exercice du pouvoir de la part de cette Maison. En somme, dans le système politico-religieux qui englobe toutes les Maisons et les hiérarchise, les rituels rendus aux ancêtres constituent un discours visant à perpétuer les droits, le rang, les propriétés matérielles d'une Maison particulière dont le caractère permanent (comme institution) est matérialisé, autour du patio privé et de la place publique, par les édifices auxquels sont en général associés ces rituels. D’un point de vue archéologique, plus vastes sont les segments sociaux auprès desquels une entité sociale légitime sa suprématie, plus les édifices particuliers associés à ces rituels sont visibles et les environnements où ils sont construits, accessibles.

\section{CONCLUSION}

La translation spatiale vers la place publique des marqueurs matériels du sacré incarnant l'institution durable de la Maison fonde le fonctionnement politique des cités mayas. Cette stratégie topologique illustre la porosité de la frontière entre espaces publics et privés, un continuum formel et fonctionnel. Reproduites de la simple maison aux complexes palatiaux jusqu'au templepyramide, les cérémonies publiques sont ancrées dans les structures constitutives de l'unité d'habitat, c'est-à-dire les relations sociales, les expériences, les normes socio-culturelles de la vie quotidienne du groupe domestique (Inomata 2006). En s'appuyant sur les fondements idéologiques et charismatiques du pouvoir, Inomata $(2000,2006)$ considère que la capacité d'exercice du pouvoir réside dans les moyens mis en œuvre par les rois mayas pour se mettre en scène, pour se rendre visibles de tous par le biais d'actes « théâtralisés ", d'actes de performance, ancrés dans les pratiques et répétés dans les interactions sociales de la maisonnée. Il n'y aurait peut-être pas plus qu'une différence d'échelle entre les rituels (ou les affaires) privés et les rituels (ou les affaires) publics (Bazy et al. 2010 ; Michelet et al. 2010). Autrement dit, la frontière entre vie publique et vie privée est fragile, les deux sphères s'interpénétrant. Le passage de l'espace social le plus petit l'espace domestique - à la société dans son ensemble s'effectue par une sorte d'emboîtements successifs. Ainsi, la frontière n'est pas le seul type d'interaction entre ces deux catégories d'espaces, l'emboîtement est une interaction par inclusion du petit dans le grand. Ce type d'interaction relie les espaces par une transformation scalaire. L'homothétie peut s'expliquer par le concept emic maya qui ne différenciait pas fondamentalement les principes de la communauté (la cité) de ceux de la famille étendue ou de la Maison. L'ajaw classique correspond à une conception de l'autorité qui valait autant pour le souverain que pour le père de famille ou le chef de maison. Ce concept expliquerait la nature fractale 
des établissements urbains mayas; il existe une certaine forme de continuité architecturale entre les espaces civico-cérémoniels publics et les ensembles résidentiels privés (voir Brown et Witschey 2003).

La frontière entre espaces publics et privés est donc non seulement un objet spatial en mouvement au gré des histoires particulières à chaque cité, mais cette frontière entre lieu public et lieu privé est fragile. La vie privée et la vie publique s'interpénètrent, le public est perçu comme supérieur, car il renvoie au pouvoir central, mais il implique le privé jusqu'au cœur créant ainsi une frontière épaisse entre ces deux catégories d'espaces. La stratégie topologique visant à projeter les éléments symboliques qui perpétuent les propriétés matérielles et immatérielles de la Maison rend possible, simultanément et dans le même lieu, deux lectures de l'espace. À première vue, telle maison privée peut être considérée comme un lieu public dès lors que l'identité politique du groupe social qui y habitait s'est attachée à la place publique sous la forme de multiples points remarquables dans ce dernier espace. Inversement, une place publique peut être considérée comme privée, dès lors que s'y sont attachées des différences et des affinités constituées en identité politique.

Ainsi, la dichotomie entre place publique et espaces privés doit être dépassée. Le régime dynastique maya classique perpétue le mode de reproduction familiale de la Maison ignorant ainsi la coupure entre le public et le privé (Bourdieu 1997). $\mathrm{Au}$ même titre que les monarchies européennes jusqu'au XVII ${ }^{\mathrm{e}}$ siècle, le régime dynastique maya classique révèle les ambiguïtés d'un système de gouvernement qui mêle le domestique et le politico-religieux, le pouvoir privé (dominium) et la puissance publique (imperium), le palais et la place publique, la « maison du roi » et la « raison d'État» (voir Bourdieu 1997). Cette dernière relation dialectique est peut-être plus appropriée, moins anachronique, pour exprimer l'ambiguïté du domestique devenu politique et du rituel familial devenu religion officielle. *

* Manuscrit reçu en juin 2013, accepté pour publication en septembre 2013.

\section{NoTES}

Cet article est une version modifiée d'une intervention réalisée dans le cadre du Groupe d'Enseignement et de Recherche Maya (GERM) rassemblant les laboratoires Ethnologie et sociologie comparative (LESC) et Archéologie des Amériques (ArchAm). Pour son invitation et relecture, je remercie chaleureusement Aurore Monod-Becquelin, mais aussi Pierre Becquelin et Cédric Becquey. Cette recherche connaît un nouvel élan cette année grâce à la bourse postdoctorale accordée par la Fondation Fyssen. Enfin, j'ai une pensée toute particulière pour Juan Antonio Valdés avec qui j'ai eu l'occasion de partager quelques discussions. Je lui dois de nombreux apports et conseils et je me rappelle qu'il fut l'un des rapporteurs de ma thèse doctorale.

1. Ce thème de «frontière épaisse », autour duquel le Groupe d'Enseignement et de Recherche Maya (GERM) a développé sa réflexion - publiée dans la revue électronique Atelier d'anthropologie [http://ateliers.revues.org/9169] - est une notion qui intéresse aussi les sciences de l'espace social (Lévy 2008, 2010) dont nous nous sommes largement inspiré afin de mieux l'appréhender dans le contexte des cités mayas classiques. 
2. Les régularités associées à un environnement social (Bourdieu 2000, p. 256).

3. La matrice des comportements individuels, qui permet à un individu d'une société donnée de se mouvoir dans le monde social et de l'interpréter d'une manière qui, d'une part, lui est propre et, d'autre part, est commune aux membres du groupe social auquel il appartient (ibid.).

4. Le principe d'abondance, testé avec succès, stipule que, dans les sites mayas, les petits monticules, du fait de leur fréquence, représentent les vestiges d'unités résidentielles (Thompson 1886 ; Haviland 1966 ; Willey et al. 1965) : il permet d'assigner une fonction résidentielle, sans fouilles, aux groupes à patio. Ce principe peut être étendu à la composante monumentale des cités si l'on reconnaît qu'elle est constituée d'une plus grande quantité d'unité-patios que d'unité-places.

5. À Copán par exemple, la Sépulture VIII-6 marque le dispositif de réception de la Structure 9N-82 (Sanders 1986) et la Sépulture XLII-6 est placée dans l'axe de la Structure 8L-87 (Ashmore 1991, p. 215).

\section{RÉFÉRENCES CITÉES}

Andrews E. Willys et Barbara W. FASH

1992 "Continuity and change in a royal Maya residential complex at Copán », Ancient Mesoamerica, 3 (1), pp. 63-88.

ARnAuld Marie-Charlotte

2001 "La "Casa Grande" : evolución de la arquitectura del poder del Clásico al Posclásico », in Andrés Ciudad Ruiz, María Josefa Iglesias Ponce de León et María del Carmen Martínez Martínez (éd.), Reconstruyendo la ciudad maya : el urbanismo en las sociedades antiguas, Sociedad Española de Estudios Mayas, Madrid, pp. 363-401.

ARnauld Marie-Charlotte et Dominique Michelet

2004 "Nature et dynamique des cités mayas", Annales: Histoire, Sciences Sociales, 2004 (1), pp. 73-108.

2010 "Casas monumentales de Río Bec, raíz del particularismo regional : variantes y variaciones ", in Aurore Monod-Becquelin, Alain Breton et Mario Humberto Ruz (éd.), Figuras mayas de la diversidad, Centro Peninsular en Humanidades y Ciencias Sociales (CEPHCIS), UNAM, Mérida, pp. 409-431.

Arnauld Marie-Charlotte, Véronique Breuil-Martínez et Erick Ponciano A.

2004 «La ciudad real», in Marie-Charlotte Arnauld, Véronique BreuilMartínez et Erick Ponciano (éd.), La Joyanca, ciudad Maya del noroeste de Petén (La Libertad, Guatemala), Centro de Estudios Mexicanos y Centroamericanos (CEMCA), Mexico-Guatemala, pp. 95-124.

AsHMORE Wendy

1991 «Site-planning and directionality among the ancient Maya », Latin American Antiquity, 2 (3), pp. 199-226.

2009 «Biography of place at Quirigua, Guatemala », in Brenda Bowser et Nieves Zedeño (éd.), The archaeology of meaningful places, University of Utah Press, Salt Lake City, pp. 15-31. 
BAZY Damien

2010 Relation entre place publique et espace privé dans les cités mayas des Basses Terres centrales et meridionales du Préclassique au Classique terminal, thèse de doctorat, Université Paris 1, Paris.

2011 «Modalidades y dinámicas de relación entre facciones políticas desde una perspectiva arquitectónica en Piedras Negras, Petén, Guatemala : el dualismo político », Trace, 59, pp. 59-73.

2013 Relation entre espaces publics et privés dans les sites mayas des Basses Terres centrales et méridionales du Préclassique au Classique terminal, coll. «British Archaeological Reports, International serie 2546/Paris monographs in American archaeology 33 », Archaeopress, Oxford.

BAzy Damien, Juan Antonio VAldés et Marie-Charlotte ARNAuld

2010 «El templo doméstico y la casa política : de rituales privados a rituales públicos en centros mayas clásicos », in Andrés Ciudad Ruiz, María Josefa Iglesias Ponce de León et Miguel Sorroche (éd.), El ritual en el mundo maya: de lo privado a lo público, Publicaciones de la Sociedad Española de Estudios Mayas (SEEM), 9, Grupo de Investigación Andalucía-América, Centro Peninsular en Humanidades y Ciencias Sociales (CEPHCIS)/ UNAM, Madrid, pp. 181-201.

BECKER Marshall J.

1979 «Priests, peasants and ceremonial centers : the intellectual story of a model », in Norman Hammond et Gordon R. Willey (éd.), Maya archaeology and ethnohistory, University of Texas Press, Austin, pp. 1-20.

Berdoulay Vincent, Paulo C. Da Costa Gomes et Jacques Lolive

2004 "L'espace public, ou l'incontournable spatialité de la politique », in Vincent Berdoulay, Paulo C. da Costa Gomes et Jacques Lolive (éd.), L'espace public à l'épreuve : régressions et émergences, Maison des sciences de l'homme d'Aquitaine, Pessac.

BLANTON Richard E.

1986 Monte Albán : settlement patterns at the ancient Zapotec capital, Academic Press, New York.

BOURDIEU Pierre

1997 «De la Maison du Roi à la raison d'État », Actes de la recherche en sciences sociales, 118 , pp. 55-68.

2000 Esquisse d'une théorie de la pratique, Éditions du Seuil, Paris.

BROwn Clifford T. et Walter R.T. WitscheY

2003 "The fractal geometry of ancient Maya settlement », Journal of Archaeological Science, 30 (12), pp. 1619-1632.

Christie Jessica J.

2003 « The tripartite layout of rooms in Maya elite residences : symbolic centering, ritual mediating, and historical governing », in Jessica J. Christie (éd.), Maya palaces and elite residences : an interdisciplinary approach, University of Texas Press, Austin, pp. 291-314. 
Demarest Arthur A.

1992 «Ideology in ancient Maya cultural evolution », in Arthur Demarest et Geoffrey Conrad (éd.), Ideology and the evolution of Precolumbian civilization, University of New Mexico Press, Albuquerque, pp. 135-157.

DESCLÉs Jean-Pierre

2002 "Categorization : a logical approach to a cognitive problem », Journal of Cognitive Science, 3 (2), pp. 85-137.

2006 "Opérations métalinguistiques et traces linguistiques », in Dominique Ducard et Claudine Normand (éd.), Antoine Culioli, Un homme dans le langage, Éditions Ophrys, Paris, pp. 41-67.

2012 « Du trimorphe aux frontières quasi topologiques », Ateliers d'anthropologie [ http://ateliers.revues.org/9172, consulté le 08/03/2013].

DesCLÉs Jean-Pierre et Zlatka GuENTCHÉvA

2010 «Quasi Topological Representations (QTR) of spatial places and spatio-temporal movements in natural languages », in Giovanna Marotta, Allessandro Lenci, Linda Meini et Francesco Rovai (éd.), Space in language : proceedings of the Pisa international conference, Edizioni ETS, Pise, pp. 213-233.

Fash Barbara, William Fash, Sheree Lane, Rudy LaRios, Linda Schele, Jeffrey StomPeR et David STUART

1992 «Investigations of a Classic Maya council house at Copán, Honduras », Journal of Field Archaeology, 19 (4), pp. 419-442.

FASH William

2005 "Toward a social history of Precolumbian civilization», in E. Wyllys Andrews et William L. Fash (éd.), Copán : the history of an ancient Maya kingdom, School of American Research Advanced Seminar Press, coll. "School of American Research Advanced Seminar series », Santa Fe/Oxford, pp. 73-101.

GeERTz Clifford

1980 Negara : the theatre state in nineteenth-century Bali, Princeton University Press, Princeton.

GODELIER Maurice

2004 «Filiation, descendance (première composante) », in Métamorphose de la parenté, Éditions Fayard, Paris.

HANKs William F.

1990 Referential practice : language and lived space among the Maya, University of Chicago Press, Chicago/Londres.

HARrison Peter D.

1970 The Central Acropolis, Tikal, Guatemala : a preliminary study of the functions of its structural components during the Late Classic period, thèse de doctorat, Université de Pennsylvanie, Philadelphie. 
Haviland William A.

1966 Maya settlement patterns : a critical review, Middle American Research Institute, Tulane University Publication 26, Nouvelle-Orléans, pp. 21-47.

HiLlier Bill et Juliette Hanson

1984 The social logic of space, Cambridge University Press, Londres.

INOMATA Takeshi

1997 "The last day of a fortified classic Maya center : archaeological investigations at Aguateca, Guatemala », Ancient Mesoamerica, 8 (2), pp. 337-351.

2000 "Kings people : Classic Maya courtiers in comparative perspective », in Takeshi Inomata et Steve Houston (éd.), Royal courts of the ancient Maya, I. Theory, comparison, and synthesis, Westview Press, Boulder, pp. 27-53.

2001 "The Classic Maya palace as political theatre », in Andrés Ciudad Ruiz, María Josefa Iglesias Ponce de León et María del Carmen Martínez Martínez (éd.), Reconstruyendo la ciudad Maya : el urbanismo en las sociedades antiguas, Sociedad Española de Estudios Mayas, Madrid, pp. 341-361.

2006 «Plazas, performers, and spectators : political theaters of the Classic Maya », Current Anthropology, 47 (5), pp. 805-842.

Inomata Takeshi, Daniela Triadan, Erick Ponciano, Estela Pinto, Richard E. Terry et Markus EBERL

2002 «Domestic and political lives of Maya elites : the excavation of rapidly abandoned structures at Aguateca, Guatemala », Latin American Antiquity, 13 (3), pp. 305-331.

InOMATA Takeshi et Steve Houston (éd.)

2000-2001 Royal courts of the ancient Maya, 2 vol., Westview Press, Boulder.

JoYCE Rosemary A.

2001 «Planificación urbana y escala social : reflexiones sobre datos de comunidades clásicas en Honduras », in Andrés Ciudad Ruiz, María Josefa Iglesias Ponce de León et María del Carmen Martínez Martínez (éd.), Reconstruyendo la ciudad Maya : el urbanismo en las sociedades antiguas, Sociedad Española de Estudios Mayas, Madrid, pp. 123-136.

LEMONNIER Eva

2006 La structure de l'habitat du site maya classique de la Joyanca (Petén NordOuest, Guatemala) dans son environnement local, thèse de doctorat, Université Paris 1, Paris.

LÉVI-STRAuss Claude

1979 «L'organisation sociale des Kwakiutl», in La voie des masques, Éditions Plon, Paris.

LÉvy Jacques

2008 «Topologie furtive», EspacesTemps.net

[http://espacestemps.net/document4543html, consulté le 14/03/2011]. 
LÉvy Jacques et Michel Lussault (éd.)

2003 Dictionnaire de la géographie et de l'espace des sociétés, Éditions Belin, Paris.

LiENDo STUARDo Rodrigo

2003 «Access patterns in Maya royal precincts », in Jessica Joyce Christie (éd.), Maya palaces and elite residences : an interdisciplinary approach, University of Texas Press, Austin, pp. 184-203.

\section{Marcus Joyce}

1983 "Lowland Maya archaeology at the crossroad", American Antiquity, 48 (3), pp. 454-488.

\section{MarTin Simon}

2000 "Court and realm : architecture signatures in the Classic Maya Southern Lowlands ", in Takeshi Inomata et Stephen D. Houston (éd.), Royal courts of the ancient Maya, I. Theory, comparison and synthesis, Westview Press, Boulder, pp. 168-194.

\section{McAnany Patricia A.}

1995 Living with the ancestors : kinship and kingship in ancient Maya society, University of Texas Press, Austin.

1998 "Ancestor and the Classic Maya built environment», in Stephen D. Houston (éd.), Function and meaning in Classic Maya architecture, Dumbarton Oaks Research Library and Collection, Washington, pp. 271-298.

McAnany Patricia A. et Shannon Plank

2000 "Perspective on actors, gender roles, and archiecture at Classic Maya courts and household », in Takeshi Inomata et Stephen D. Houston (éd.), Royal courts of the ancient Maya, I. Theory, comparison and synthesis, Westview Press, Boulder, pp. 84-129.

Michelet Dominique, Philippe Nondédéo, Grégory Pereira, Julie Patrois, MarieCharlotte Arnauld et Alfonso LaCAdEna

2010 "Rituales en una sociedad "sin" reyes : el caso de Río Bec y del Edificio A (5N2) en particular ", in Andrés Ciudad Ruiz, María Josefa Iglesias Ponce de León et Miguel Sorroche (éd.), El ritual en el mundo maya : de lo privado a lo público, Publicaciones de la Sociedad española de estudios Mayas (SEEM), 9, Grupo de Investigación Andalucía-América, Centro Peninsular en Humanidades y Ciencias Sociales (CEPHCIS), UNAM, Madrid, pp. 153-180.

\section{Monod-BecQuelin Aurore}

2012 «Introduction », Ateliers d'anthropologie [http://ateliers.revues.org/9170, consultéle 15/05/2013]. 
MoORE Jerry D.

1992 «Pattern and meaning in prehistoric Peruvian architecture: the architecture of social control in the Chimu state», Latin American Antiquity, 3 (2), pp. 95-113.

1996a Architecture and power in the ancient Andes : the archaeology of public buildings, Cambridge University Press, Cambridge.

1996b "The archaeology of plazas and the proxemics of ritual : three Andean traditions », American Anthropologist, 98 (4), pp. 789-802.

NONDÉDÉo Philippe

2003 L'évolution des sites mayas du sud de l'État du Campeche, Mexique, coll. «British Archaeological Reports, International serie 1171/Paris monographs in American archaeology 12 », Archaeopress, Oxford.

Peterson Christian E. et Robert D. Drennan

2005 «Communities, settlements, sites, and surveys : region-scale analysis of prehistoric human interaction ", American Antiquity, 70 (1), pp. 5-30.

Pierrebourg Fabienne de

1999 L'espace domestique maya: une étude ethnoarchéologique au Yucatán, coll. "British Archaeological Reports, International serie 764/Paris monographs in American archaeology 3 ", Archaeopress, Oxford.

Pierrebourg Fabienne de, Marie-Charlotte Arnauld, Helios Figuerola, Jean-Michel Hoppan, Olivier Le Guen et Perla Petrich

2012 «L'habitation maya », Ateliers d'anthropologie [http://ateliers.revues.org/ 9237, consulté le 15/04/2013].

SANDERS William T.

1986 Excavaciones en el área urbana de Copán, tome I, Secretaria de Cultura y Turismo, Instituto Hondureño de Antropología e Historia, Tegucigalpa.

THOMPSON Edward H.

1886 "Archaeological research in Yucatan », Proceedings of the American Antiquarian Society, 4, pp. 258-254.

TOBAR Telma et Breitner GonZÁLEZ

2007 "La Acrópolis de Nakum : un análisis espacial y volumétrico », in Juan Pedro Laporte, Bárbara Arroyo et Héctor E. Mejía (éd.), $X X$ Simposio de investigaciones arqueológicas en Guatemala, 2006, Museo Nacional de Arqueología y Etnología, Guatemala, pp. 644-658.

TURNER Victor

1990 Le phénomène rituel : structure et contre-structure, PUF, Paris [1969].

VALDÉs Juan Antonio

2001 "Palaces and thrones tied to the destiny of the royal courts in the Maya lowlands ", in Takeshi Inomata et Stephen D. Houston (éd.), Royal courts of the ancient Maya, II. Data and case studies, Westview Press, Boulder, pp. 138-164.

2005 El periodo clásico en Uaxactun, Guatemala : arqueología en el centro de Petén, Guatemala, Instituto de Investigaciones Históricas, Antropológicas 
y Arqueológicas, Escuela de Historia, Universidad de San Carlos de Guatemala, Guatemala.

Webster David et Stephen D. Houston

2004 "Piedras Negras : the growth and decline of a Classic Maya court center », in William T. Sanders, Alba G. Mastache et Robert H. Cobean (éd.), Urbanism in Mesoamerica, Vol. I, Instituto Nacional de Antropología e Historia/The Pennsylvania State University, Mexico, pp. 427-450.

Willey Gordon R., William R. Bullard Jr., John B. Glass et James C. Gifford

1965 "Prehistoric Maya settlements in the Belize valley », Peabody Museum of Archaeology and Ethnology Papers, 54, Harvard University, Cambridge.

ŹralKa Yaroslaw et Bernard Hermes

2012 «Great development in troubled times : the Terminal Classic at the Maya site of Nakum, Petén, Guatemala », Ancient Mesoamerica, 23 (1), pp. 161-187.

Źralka Yaroslaw, Bernard Hermes et Zoila CALDERón

2007 «El periodo Clásico terminal en Nakum : el momento de mayor auge constructivo y aumento poblacional de un sitio maya del noreste de Petén » in Juan Pedro Laporte, Bárbara Arroyo et Héctor E. Mejía (éd.), XX Simposio de investigaciones arqueológicas en Guatemala, 2006, Museo Nacional de Arqueología y Etnología, Guatemala, pp. 513-537. 